\title{
A review: controlling the propagation of surface acoustic waves via waveguides for potential use in acoustofluidics
}

\author{
Jiyang MEI* and James FRIEND* \\ * Medically Advanced Devices Laboratory, \\ Department of Mechanical and Aerospace Engineering, Jacobs School of Engineering \\ Department of Surgery, School of Medicine \\ University of California San Diego, 9500 Gilman Dr, La Jolla, CA 92093, USA \\ E-mail: jfriend@eng.ucsd.edu
}

Received: 16 August 2019; Revised: 9 October 2019; Accepted: 28 October 2019

\begin{abstract}
This paper presents a review of waveguides on lithium niobate for surface acoustic waves (SAWs), including in particular the classic literature on the topic with the intent of renewing interest in them in the context of potential applications in the burgeoning discipline of micro to nano-scale acoustofluidics. From the fundamentals of the piezoelectric effect we describe interdigital electrodes and how they generate acoustic waves, consider focusing interdigital electrodes as a simple means of laterally confining the acoustic energy propagating across a substrate, and then quickly move to waveguiding structures that provide confinement by defining either a region of slow wave velocity or a physically isolated structure. The ability to steer acoustic waves using these waveguides is considered. The many analytical, computational, and experimental tools devised by past investigators to design them are discussed in detail, as are the relative advantages and disadvantages of the waveguide designs considered over the years.
\end{abstract}

Keywords : Piezoelectric material, Surface acoustic wave (SAW), Lithium niobate (LN), Anisotropy, Waveguides, Dispersion relation, Acoustofluidics

\section{Introduction}

\subsection{Electro-acoustic waves and piezoelectric materials}

Experiments involving vibration in manipulation of particles date back to the late 1700's, notably the work of Chladni (1787), Faraday (1831), and Kundt (1866). Soon after these contributions, the phenomenon of surface acoustic wave (SAW) propagation on a substrate was first reported by Lord Rayleigh (1885). Wave generation was mostly limited to bulk acoustic waves (BAWs) until the advent and broad acceptance of interdigital transducers (IDTs) that enabled high frequency SAW.

Microscale acoustofluidic actuation relies on the well-known piezoelectric effect, which generates electric charge upon the application of mechanical stress in certain materials. Electric dipole moments are formed from the molecules in a piezoelectric material when their crystal structure is altered by mechanical strain. The macroscopic effect is of significance due to charge accumulation for each repeated unit in the crystal (Curie et al., 1881). This process is also reversible: the application of an electric field responds to the atomic dipoles to create strain, through electrostatic interaction. Piezoelectric crystals can therefore act as electromechanical transducers.

Actuation by SAW avoids the limitations that plague conventional ultrasonic methods: relatively lower frequency, smaller accelerations, and difficulty of device miniaturization. When traveling in an optimal substrate, SAWs typically can produce surface displacement up to $1 \mathrm{~nm}$ at high frequency, which leads to large acceleration. Research utilizing ultrasonics and acoustics to manipulate fluids at the microscale-microscale acoustofluidics - has seen exponential growth, with extensive reviews by Friend et al. (2011), Ding et al. (2013), and more recently Destgeer et al. (2015) and Connacher et al. (2018). 
The most popular materials that are used to make SAW devices include quartz, lithium tantalate (LT) $\mathrm{LiTaO}_{3}$ and lithium niobate ( $\mathrm{LN}) \mathrm{LiNbO}_{3}$. Others include gallium arsenide (GaAs), cadmium sulfide (CdS), zinc oxide ( $\mathrm{ZnO}$ ), lithium tetraborate $\left(\mathrm{Li}_{2} \mathrm{~B}_{4} \mathrm{O}_{7}\right)$, and lanthanum gallium silicate $\left(\mathrm{La}_{3} \mathrm{Ga}_{5} \mathrm{SiO}_{12}\right)$ (Thurston et al., 1998). Due to the crystalline structure of these materials, the type of wave generated is strongly dependent on the choice and orientation of the material as discussed above. Lithium niobate has been the preferred material for devices requiring high efficiency, as it possesses a relatively large electromechanical coupling coefficient $K^{2}=2 \Delta v / v=2\left(v_{f}-v_{m}\right) / v_{f}$ where $v_{f}$ is the SAW velocity upon a substrate free of any electroding (i.e., open-circuit) and $v_{m}$ is the SAW velocity along a short-circuited surface. Of the various cuts possible, the $131^{\circ} Y$-rotated cut for $X$-propagating SAW in LN was found to have the highest electromechanical coupling coefficient for SAW (Slobodnik et al., 1970), relative to other cuts in LN and other choices of single-crystal piezoelectric media. In 1976, Shibayama et al. conducted experiments to determine the optimum rotated $Y$-cut of LN to generate "true" SAWs and concluded that the $127.86^{\circ} Y$-rotated choice for $X$-propagating SAW in lithium niobate (128 YX LN) reduced the generation of other parasitic waves and retained most of the electromechanical coupling and low insertion loss observed in the $131^{\circ} Y$-rotated cut of LN.

\subsection{Generating SAW on lithium niobate}

Interdigital transducers are one of the most widely used devices that generate and detect SAWs. The first and simplest IDTs (White et al., 1965) consisted of straight rectangular metal bars-fingers-deposited on the surface of a piezoelectric substrate. Alternately connected on their end to common electrodes or bus bars, the fingers appear as depicted in Fig. 1. An array of electric fields of alternating direction is created between the transducer finger pairs, and thus in turn induces alternating regions of compression and tension in the substrate. Each finger pair therefore produces displacement in the substrate that oscillates with the electric field to produce SAW. The wavelength of the resulting SAWs ( $\left.\lambda_{\text {SAW }}\right)$ is defined by the periodicity of the finger pairs, such that the finger width and the spacing between each two fingers are both $\lambda_{\mathrm{SAW}} / 4$. As a result, the center frequency $\left(f_{r}=\omega / 2 \pi=v_{R} / \lambda_{\mathrm{SAW}}\right)$ of a given device is determined by the choice of substrate, propagation direction and IDT design, particularly the finger width and spacing. This corresponds to the resonant frequency of a SAW device, where the power density and surface displacement are maximized (Dentry et al., 2014; White et al., 1965). Over the years, more advanced designs have been devised (Campbell, 1998), incorporating apodization of the fingers (Haydl, 1972), dual finger pairs (Campbell, 1998), tapered (Lamperski, 1992) and stepped (or "quasi-slanted") fingers (Bausk et al., 2004), chevron and slanted (Bausk et al., 2004) fingers, and special arrangements to facilitate broader bandwidth such as the chirp IDT (Fall et al., 2017) or internal reflectors to form a unidirectional SAW in the so-called SPUDT (single-phase unidirectional transducer) (Shui et al., 2002) and its variants.

In IDT design, the substrate thickness is of importance, determining the minimum frequency of the SAW. The Rayleigh SAWs are recognized to travel 4-5 $\lambda$ into the substrate, while the most commonly used LN wafers are 500 $\mu \mathrm{m}$ thick, forcing a rough threshold of the frequency suitable to form Rayleigh SAW of about $40 \mathrm{MHz}$. Acoustic waves of large wavelength, corresponding to frequencies below this value, can penetrate all the way through the substrate and dissipate energy to the other side in the form of spurious Lamb waves alongside Rayleigh SAW, which will inevitably affect the wave propagation and device performance. This limit has been routinely violated in the literature, apparently due to poor understanding of the SAW phenomena in research groups more skilled in microfluidics or fluid dynamics, and the currently rare availability of non-contact vibration measurement techniques in most groups above $25 \mathrm{MHz}$.

The wave is diffracted as the SAW propagates through subsequent finger pairs, creating a near-field region of largely parallel wavefronts known as the Fresnel region. The far-field region, where SAW is broadly diffracted along major and minor lobes, is known as the Fraunhofer region. For design purposes, the Fresnel parameter $F=4 \lambda_{\mathrm{SAW}} D_{F} / a^{2}$ delineates the distance from the source (IDT) at which the transition is made between the near $(F<1)$ and far-field $(F>1)$ regions, where $a$ is the aperture width (shown in Fig. 1) and $D_{F}$ is the distance from the IDT edge. The number of finger pairs, $N_{p}$, of a SAW device is also an important parameter due to its effect on the quality factor $Q=f_{r} / \Delta f$ (Morgan, 2007), itself describing the efficiency of wave propagation, where $\Delta f$ is the width of the resonant peak as measured at one-half the maximum (peak) amplitude. A larger quality factor represents less loss, with typical values for SAW of 10,000 or more in $\mathrm{LN}$ in contact with air but far less when in contact with water. Further, the electromechanical coupling coefficient is inversely proportional to $N_{p}$. Depending on the application needs, there is an optimal choice for the number of IDT finger pairs $N_{p}$ that balances the quality factor $Q$ and the electromechanical coupling coefficient $K^{2} \propto 2 \Delta v / v$.

Compared to the straight IDTs described above, focused IDTs (FIDTs), can generate SAW with greater intensity and higher beamwidth compression ratios $\eta_{c}(=H / W)$, where $H$ is the $-3 \mathrm{~dB}$ transverse beamwidth and $W$ is the equivalent aperture of the FIDTs. They have been utilized in many applications, such as signal processing convolvers (Green et 
al., 1983; Nakagawa, 1991; Marynowski, 1982), storage correlators (Green et al., 1980), and time-Fourier transformers (Wilcox et al., 1985). High intensity acoustic fields can also be generated, and thus enhance the acoustic-electric effect in order to manipulate electron-hole pairs in GaAs quantum wells (Lima Jr et al., 2004).

FIDTs were first introduced by Kharusi et al. in 1972. They examined two different FIDT patterns: the circular-arc shape (see Fig. 2) and the group-velocity surface shape, which consisted of points tracked by the energy velocity vector and drawn from a fixed original point (virtual point source) as the propagation direction varied. Their results showed that both IDT designs were able to focus SAWs, but that the latter led to a superior ability to focus the SAW. Later in the 1980s, Fang et al. (1989) using an angular spectrum formulation and the uniform asymptotic method (Ursell, 1972; Budden, 1976), calculated the amplitude field of an FIDT with circular-arc design on $Y$-cut $Z$-propagating LN (YZ LN) and discovered that the acoustic energy excited was actually focused into a long, narrow region about the propagation axis (see Fig. 3). The results were verified by their experimental data.

A more general study of various IDT configurations was provided by Wu et al. (2005a) and Wu et al. (2005b). They adopted the angular spectrum of plane wave theory and combined the theoretical work with experimental results. They

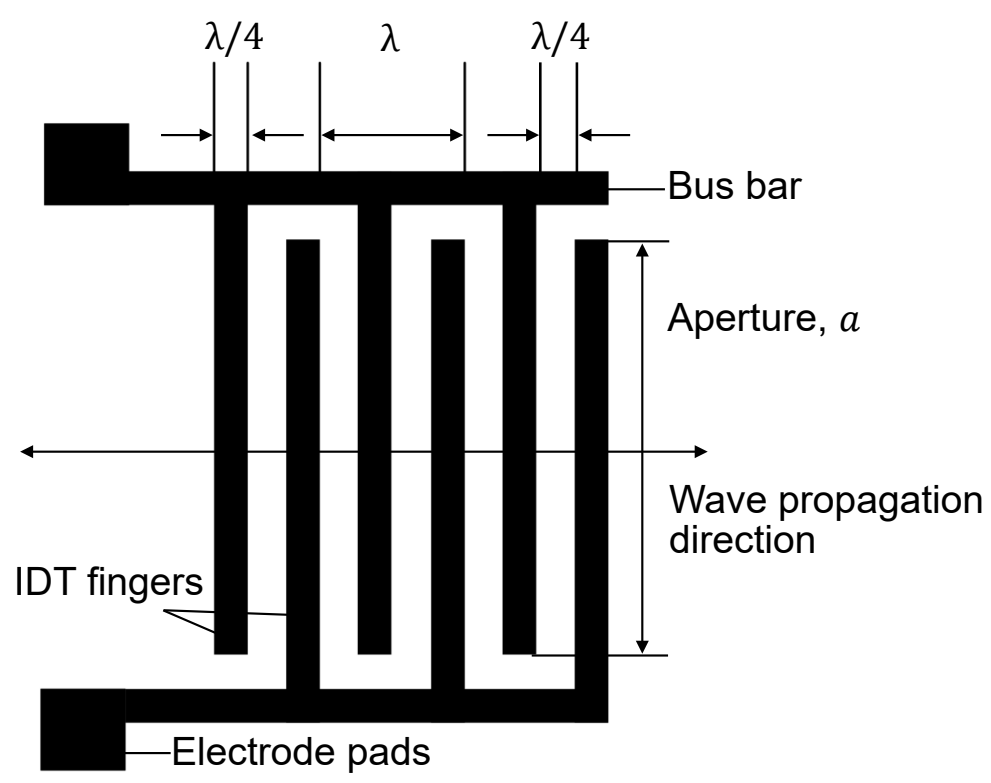

Fig. 1 A SAW device, consisting of comb-like interdigital transducers, electrode pads on a piezoelectric substrate (e.g., $128^{\circ} \mathrm{YX} \mathrm{LN}$ ). The resulting traveling wave is propagating on the $X$ axis of the $\mathrm{LN}$ and can be observed from a laser Doppler vibrometer (LDV). The periodicity of the finger pairs defines the wavelength of the resulting SAW, $\lambda_{\mathrm{SAW}}$. (Figure adapted from Connacher et al. (2018))

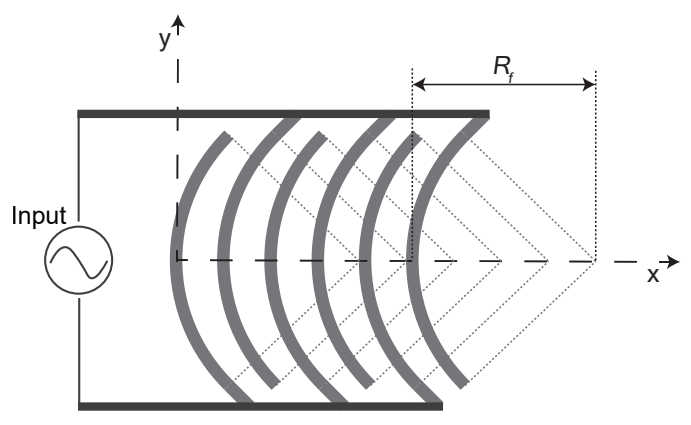

(a)

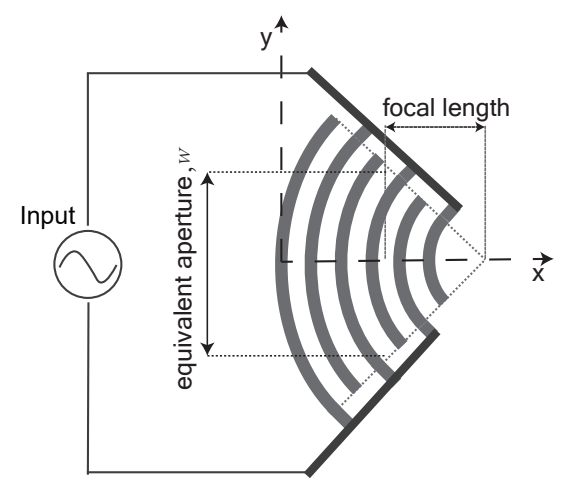

(b)

Fig. 2 Commonly used FIDTs designs: (a) conventional structure and (b) concentric structure. $R_{f}$ is curvature of transducer finger and $W$ is the equivalent aperture of transducer. It is clearly showing that the focal point of concentric design is closer to the IDTs, indicating better focusing property. (Figure adapted from Connacher et al. (2018)) 


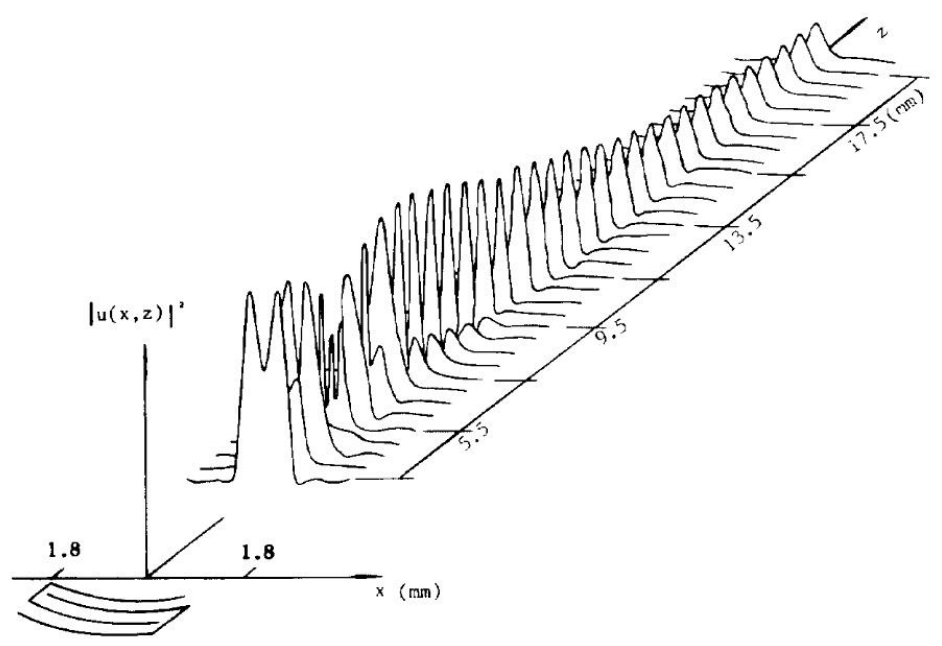

Fig. 3 Acoustic field profile of FIDT obtained by numerical integral on complex plane, showing that the acoustic energy is focused into a narrow and long region of the propagation axis. In this particular example, the acoustic energy is propagating on a YZ LN. (Figure permission acquired from Fang et al. (1989))

were thus able to conclude that conventional circular-arc FIDTs (see Fig. 2(a)) experienced reduced focusing properties as $N_{p}$ increases, while concentric-arc FIDTs (see Fig. 2(b)) displayed a stable amplitude field and better focusing characteristics. FIDTs with concentric-elliptic arcs generated a narrow, long, strong line SAW source. Their findings were further confirmed through direct visualization of the amplitude field generated by FIDTs. Shilton et al. (2008) showed SAW propagation patterns generated on $128^{\circ} \mathrm{YX} \mathrm{LN}$ surface for a straight single-phase unidirectional transducer (SPUDT), concentric-elliptic SPUDTs with various eccentricities, and a concentric circular SPUDT using scanning laser Doppler vibrometer (LDV) (see Fig. 4). Their results proved that curved IDT fingers focused SAWs while straight SPUDTs did not. Additionally, as the eccentricity increased, the SAW pattern became narrower and finally concentrated to a single focal point.

The above contributions have allowed FIDTs to be widely employed in acoustofluidic applications where their high intensity and greater beamwidth compression ratio can be utilized. Droplet translation and merging (Jung et al., 2016; Sesen et al., 2017; Sesen et al., 2014), particle concentration and mixing (Collins et al., 2016a; Collins et al., 2017), and cell sorting (Ren et al., 2015; Collins et al., 2016b) have all benefited from the use of FIDTs due to their ability to maximize the proportion of acoustic energy that results in efficient acoustic force gradients and acoustic streaming. As one advanced design of IDTs, rather than propagating forward with a fixed width along the LN substrate, FIDTs successfully manipulate SAWs to some extent, so that they converge into one narrow region or even a small spot. However, this level of manipulation is often inadequate.

Other types of IDTs, such as double electrode IDTs, slanted-finger IDTs, and chirp IDTs, which are also commonly used in the field of acoustofluidics for other purposes, are not covered in this paper.

\section{Waveguides}

\subsection{Why waveguides?}

As discussed in Section 1.1, SAW devices are usually designed so that waves propagated along the direction correspond to different rotated cuts of the material. However, materials most commonly used in acoustofluidics are anisotropic in nature due to their crystal structure. The $128^{\circ}$ YX LN, for example, can generate SAWs of the same frequency and amplitude only in one direction. Any veering from the primary propagation direction is affected by beam steering (explained later), SAW velocity or frequency, and the SAW's electromechanical coupling coefficients. Kurosawa et al. (1995) demonstrated in using SAW along both the $X$ and $Y$ axes of a $128^{\circ}$ YX LN wafer that the vibration displacement and velocity were $250 \%$ higher along the $X$ axis compared to the $Y$ axis.

Though efforts had been made to overcome the anisotropic nature of the piezoelectric substrates, as Guo et al. (2015) and Devendran et al. (2016) illustrated perpendicular IDT pairs at $45^{\circ}$ to the $X$-axis to create an orthogonal standing wave at the same frequency, the issue of beam steering remains: beam steering is lateral transport of the acoustic wave at an angle to the intended propagation direction, usually toward a specific crystallographic axis of the substrate, such as the $X$ axis in $128^{\circ} \mathrm{YX} \mathrm{LN}$ for SAW propagating at some angle with respect to this axis. The superior electromechanical coupling 

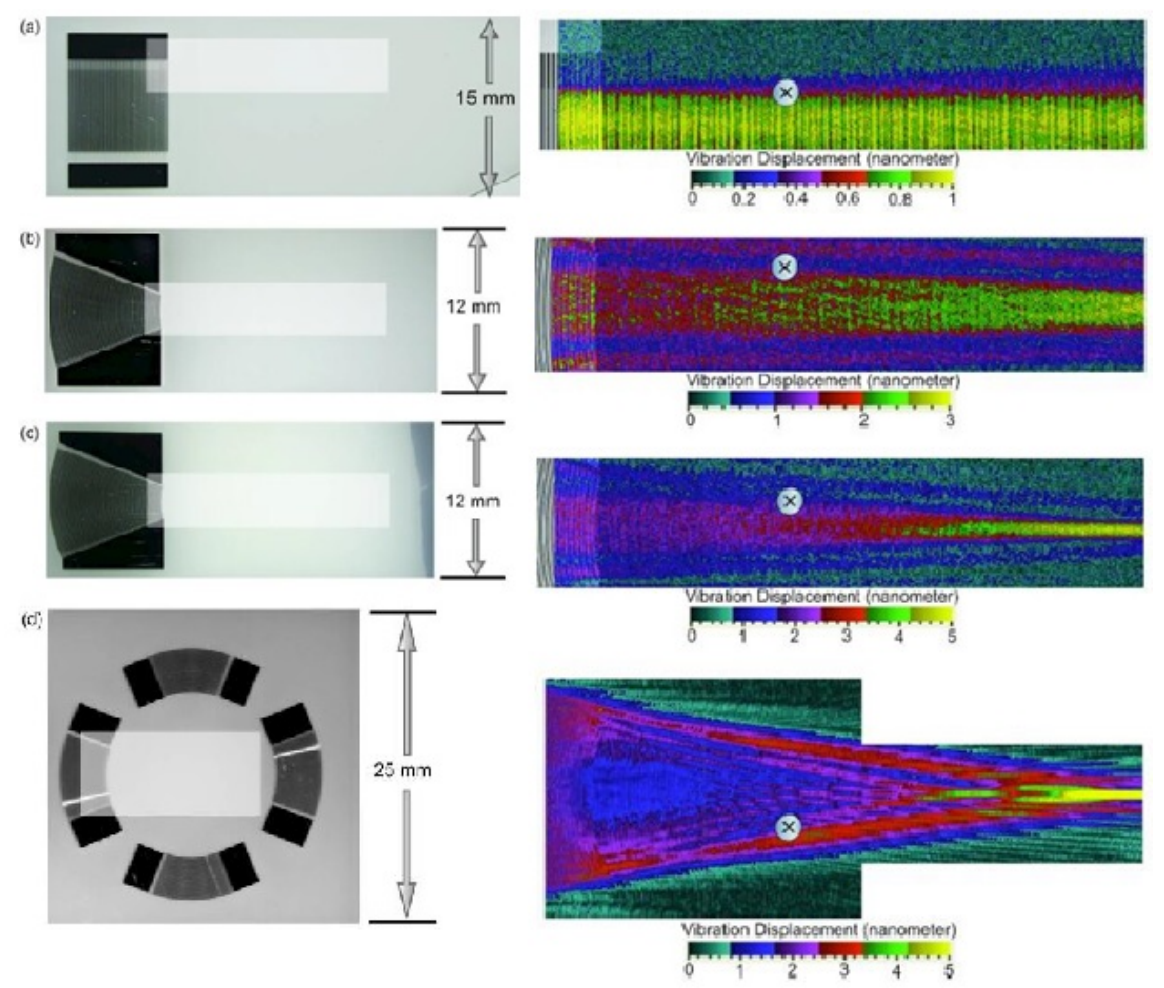

Fig. 4 Understand the distribution of SAW displacement on a LN substrate of different IDT designs at center frequency of $30 \mathrm{MHz}$ : (a) straight SPUDT (b) focusing elliptical SPUDT with an approximate eccentricity of 0.616, (c) focusing elliptical SPUDT with an approximate eccentricity of 0.831, and (d) focusing circular SPUDT. It shows that the displacement of the SAW produced by straight SPUDT is uniform on the substrate. While the the acoustic beam focuses into a single point when the eccentricity of a focusing elliptical SPUDT is high enough. (Figure adapted from Shilton et al. (2008)).

present along the $X$-axis and the unmatched wave velocities along the $X$ - and $Y$-axis are responsible for the phenomena in this case. Consequently, the design, performance characteristics and ultimately efficiency of high frequency SAW devices made using LN substrates are typically limited to the $X$ axis direction for acoustic wave propagation.

Another problem with SAWs on LN is lateral diffraction, a major constraint in current SAW devices inevitably resulting in energy dissipation along certain directions. As shown in Section 1.2, SAW propagates as largely parallel wavefronts in the near-field region (Fresnel region); while in the far-field region (Fraunhofer region) it is broadly diffracted. Though one may seek to minimize its effects by the choice of a low diffraction propagation direction, there are situations in which fluid or particle manipulation at disparate locations in a micro device may be expected. The difficulties in controlling the direction of wave propagation make these applications inefficient as well (Oliner, 1976).

However, these limitations may all be overcome by the use of waveguides for such surface waves, where the term waveguide implies a geometrical structure which confines the lateral extent of SAWs. Wave diffraction, which is one of the most important difficulties associated with wide surface wave propagation, is thereby automatically prevented as the field cross section associated with the waveguide remains constant as the wave travels. As long as the radius of the bend is not too small, the propagating wave will remain bounded to the guiding structure.

Waveguides have been widely utilized in the applications of long delay lines (Adams et al., 1972) and structural health monitoring (Raghavan et al., 2007; Siqueira et al., 2004), while in the context of acoustofluidics is absent for two reasons. In some applications, losses due to waveguides can be significant, although sometimes wide-beam surface waves on the material can be even lossier. The other reason could be the question of whether or not waveguides are worth their design and fabrication difficulty to meet current device requirements. Nevertheless, current SAW devices generally perform single functions: mixing (Rezk et al., 2012; Tseng et al., 2006), sorting (Ding et al., 2012; Schmid et al., 2014), concentration (Shi et al., 2008; Tan et al., 2009), pumping (Schmid et al., 2012; Langelier et al., 2012) and so on. When it inevitably comes to more sophisticated and multifunctional design, waveguides can be called upon, due to the ability of waveguides to confine and control the wave propagation. 


\section{Flat overlay waveguides}

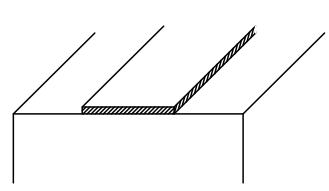

(a)

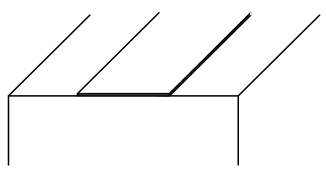

(b)

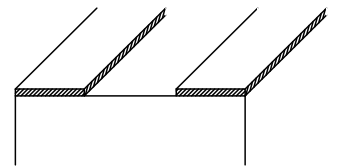

(c)

\section{Topographic waveguides}

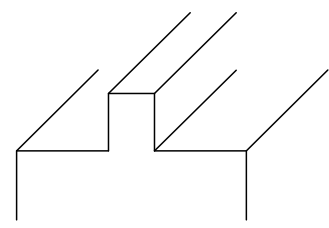

(d)

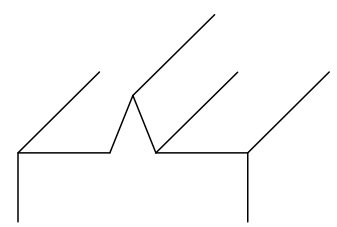

(e)

\section{Change of surface property}

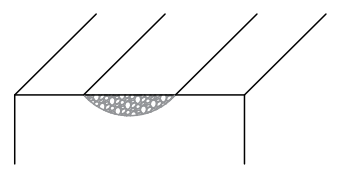

(f)

Fig. 5 Various types of waveguides for SAW. Flat overlay waveguides: (a) strip, (b) shorting-strip, (c) slot. Topographic waveguides: (d) rectangular ridge, (e) wedge. Change of surface property: (f) in-diffused or ion-implanted, (Figure redrawn from Oliner (1976))

\subsection{Types of waveguides}

There are mainly three types of waveguides (Oliner, 1976):

(1) Overlay waveguides: a thin film of another material is deposited on the substrate,

( 2 ) Topographic waveguides: a locally defined structure from the substrate surface itself,

(3) Waveguides that produces a local change of the properties of the substrate instead of its geometry.

Flat overlay waveguides may be the most well known, as they have been thoroughly analyzed and experimentally utilized (Yen et al., 1976). The central region in each case is considered the relatively low-velocity (slow) wave region, so that the SAW field will be laterally confined in the central region: any SAW exiting the slow region will be forced to speed up, thus caused to turn back into the slow region and away from the fast region. In the strip waveguide (see Fig. 5(a)), the strip itself is a material in which SAW travels slower than in the substrate. The shorting-strip waveguide (see Fig. 5(b)) is made of a conducting layer upon and in contact with the piezoelectric substrate. The conducting thin film short-circuits the electric field associated with the piezoelectric SAW, producing a slight decrease of the wave velocity under the strip. It is also known as the $\Delta v / v$ waveguide, as the velocity is reduced by the shorting strip in an amount directly dependent upon the electromechanical coupling, itself proportional to the $\Delta v / v$ ratio. In the slot waveguide structure (see Fig. 5(c)), the overlay strips on the sides are "faster" than the substrate, making the central region "slower". More details on thin-film waveguides will be discussed in Section 2.3.

Topographic waveguides guide the waves by reducing the straining forces acting on the material (Ash et al., 1969). It is also pointed out in the literature that such guiding structures have no counterpart in electromagnetic waveguides. The rectangular ridge waveguide (see Fig. 5(d)) includes two dominant modes: symmetric and antisymmetric. The symmetric mode possesses no dispersion and is also called the pseudo-Rayleigh mode as its displacement field resembles a slice out of a Rayleigh SAW (Yen et al., 1975; McCoy et al., 1963). By contrast, the better-known antisymmetric mode exhibits strong dispersion (Lagasse, 1973a; Lagasse et al., 1972; Lagasse, 1973b; Lagasse, 1973c). The wedge waveguide (see Fig. 5(e)) has no dispersion for waves possessing a frequency over a certain critical value (Lagasse et al., 1972). These waveguides will be considered in greater detail in Section 2.4.

The structure in Fig. 5(f) generally remains geometrically flat, as the properties of the substrate are altered-in the dotted region shown in the figure-by atomic in-diffusion or ion-implantation to produce measurable changes in the SAW 
Table 1 Summary of the properties of different types of waveguides

\begin{tabular}{|c|ccccc|}
\hline Type of waveguide & Field Confinement & Amount of Slowing & Dispersion & Loss & Versatility \\
\hline Strip & moderate & moderate & moderate & medium & moderate \\
Shorting-strip & weak & small & low & medium & low to moderate \\
Slot & weak to moderate & small to moderate & low & low to medium & moderate \\
In-diffused & weak & small & low & low & low to moderate \\
Topographic ridge, symmetric mode & strong to very strong & moderate to large & high & low & high \\
Topographic ridge, antisymmetric mode & very strong & small & very low & low & high \\
Topographic wedge & very strong & moderate to very large & very low to nil & low & high \\
\hline
\end{tabular}

velocity. For example. Schmidt (1975) demonstrated the diffusion of certain metals into LN produced a velocity increase of about one to two percent, while there was no apparent increase in loss due to the in-diffusion process. Such lowloss modification of the substrate is helpful for some applications in acoustofluidics where high power SAW is typically necessary. The in-diffused and ion-implanted waveguides will be discussed further in Section 2.5.

Each of the waveguide configurations above possesses unique properties, and the main features, advantages, and disadvantages of each are summarized in Table 1 (Oliner, 1976).

Over the past few years, waveguiding layers have also found use as biosensors (Xu et al., 2018; Gizeli et al., 2003). The typical structure consists of input IDTs and output IDTs both on a piezoelectric substrate. The input IDTs generates SAW propagating towards the output. With a thin dielectric material overlaid on the device, the acoustic energy is therefore confined in this guiding layer. Perturbations to the SAW during its propagation through the guiding layer, such as a change in the resonant frequency, electromechanical coupling, or wave attenuation, may be identified at the output IDTs. The presence of the thin layer, at approximately one wavelength thick, greatly increases the sensitivity of the sensor (Kabir et al., 2016; Rocha-Gaso et al., 2009). However, we focus on actuation rather than sensing, as so the ample literature in the field is set outside the scope of this review article.

\subsection{Thin film waveguides}

The concept of the thin film waveguide was first introduced by Seidel et al. (1970). They suggested that thin metal films of finite width deposited on an isotropic substrate can be used to guide elastic surface waves in the substrate. This kind of guiding structure in the use of SAW applications reduced the wave propagating velocity in the corresponding area either by its own property of being "slow" or short circuiting the electric field of the substrate, so that waves on the surface tended to be confined in the low-velocity region and propagate accordingly, as shown in Fig. 6. The guiding mechanism of thin film waveguides is analogous to the total reflection of an electromagnetic wave (e.g., light) when a dielectric thin film is placed on the substrate. The "slow" region, relative to the "fast" region, corresponds in the optical case to a strip with a higher index of refraction.

\subsubsection{Strip waveguides}

The strip waveguide involves a "slow" material (strip) and a "fast" (substrate). The wave is inclined to travel in the region where the wave velocity is lower than that in the other material. The strip is usually wide and flat, with a width-

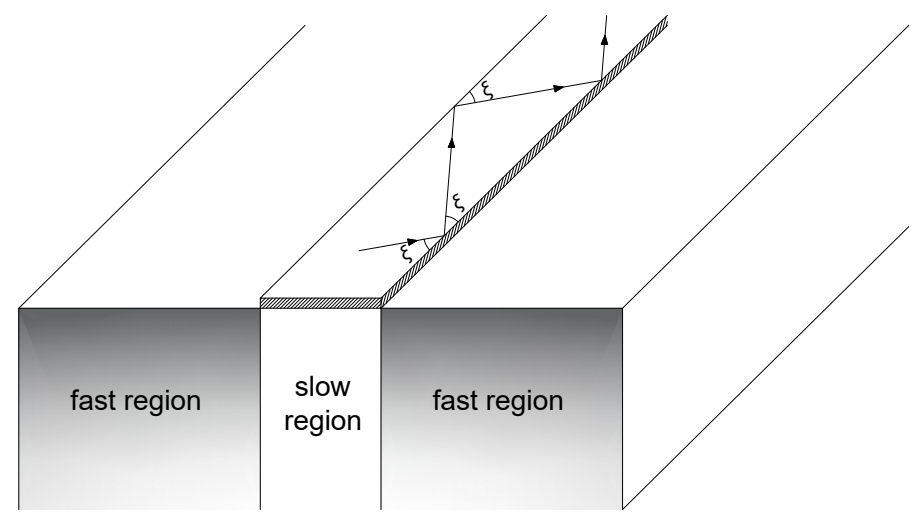

Fig. 6 Model of thin film waveguide showing wave zigzagging back and forth in the "slow central region". (Figure redrawn from Schmidt et al. (1975)) 


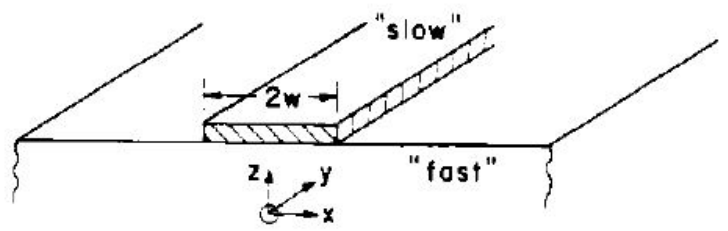

(a)

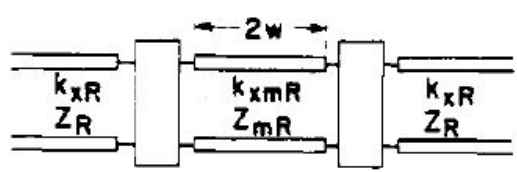

(b)

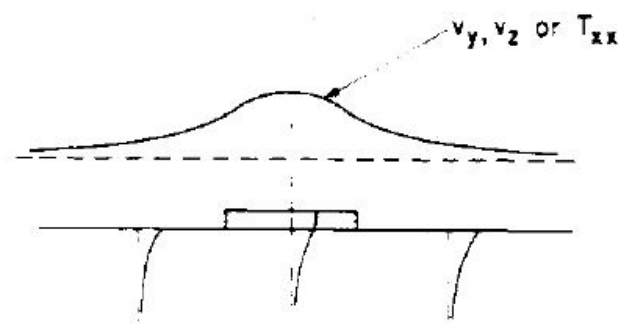

(c)

Fig. 7 The (a) geometry of a strip waveguide, consisting of an overlay of "slow" material on a "fast" substrate. (b) The transverse equivalent network for the dominant mode; $k_{x R}$ and $k_{x m R}$ are the transverse wavenumbers in the $x$ direction. (c) The transverse acoustic field behavior; $v_{x}$ and $v_{y}$ are particle velocity components and $T_{x x}$ is a stress tensor component. (Figure permission acquired from Oliner (1976))

to-thickness aspect ratio ranging from 5 to 50, and the slowing effect is obtained through mass loading of the substrate, enabling the choice of non-conductive materials for the strip. Conductive strip materials can also be used, but this tends to be associated with a different form of waveguide that principally functions by short-circuiting the piezoelectric substrate below, covered in Section 2.3.2.

One of the analytical methods in theoretically investigating the guiding performance of these waveguides is microwave network analysis (Li et al., 1977; Oliner et al., 1971). Tiersten (1969) proposed the pioneering theoretical investigation of structures which depended upon a velocity differential for guidance. The structure of a strip waveguide is replotted in Fig. 7(a). An equivalent network is derived from the approach, allowing us to view the propagation of the guided mode of the modified Rayleigh wave in the strip region which bounces back and forth from the strip sides, as depicted in Fig. 7(b). The modified Rayleigh wave is shown as the central transmission line, and the outer transmission lines represent the Rayleigh waves on the free surface. The transverse field behavior for the dominant mode is summarized in Fig. 7(c). The transverse decay along the substrate surface ( $x$ direction), simply decreases exponentially. The acoustic field substantially stretches out generally for the waveguides so that the wave is weakly bounded, which discourages sharp bends on the surface.

The dispersion behavior, variation of propagation velocity with frequency, is distinct for those waveguides described in Section 2.2. The dispersion properties of the strip waveguide are shown in Fig. 8, where the aspect ratio is defined as $G=w / t$ ( $w$ is the half width and $t$ is the thickness of the metal strip on the substrate). It can easily be inferred that the free surface Rayleigh wave is faster than that in the guiding structure. And for waveguides with a higher aspect ratio, the guided wave is slower. Furthermore, the field spreads out at low frequencies, which indicates that none of the field is in the guiding layer with energy propagation in the substrate as a Rayleigh wave. The velocity remains approximately the same as the Rayleigh wave on the substrate. When the frequency is increased, the wave velocity decreases as more of the field is confined into the "slow region". Finally, when the frequency is very high, nearly all the wave displacement field is confined to the slow region of the strip and the velocity decreases to the velocity of the Rayleigh wave in the strip material alone. The dispersion relation for the dominant guided mode is given by the lowest resonance value found from 


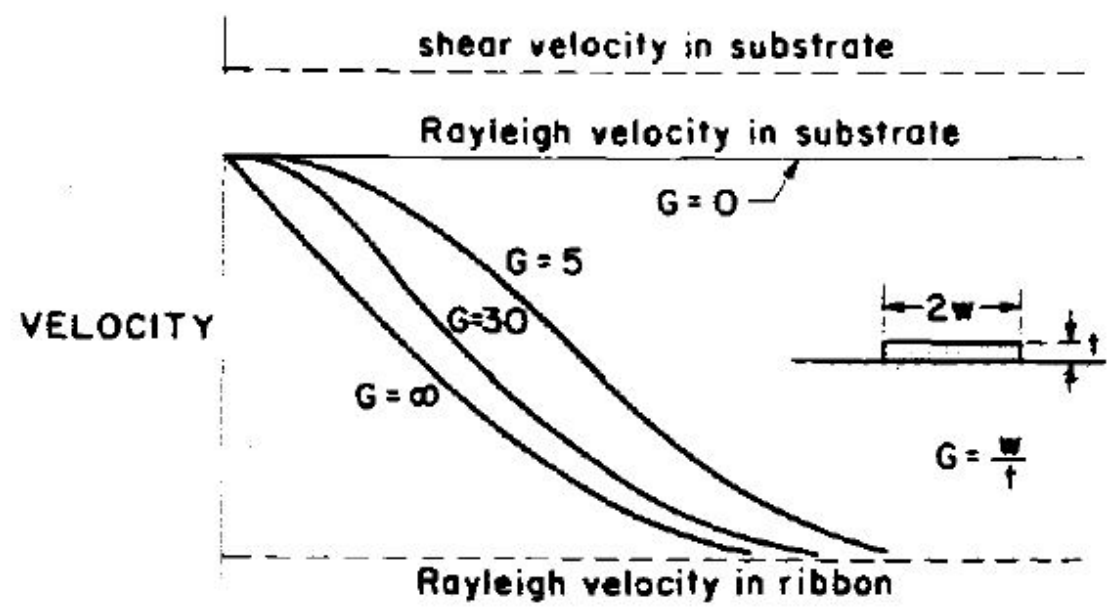

\section{FREQUENCY}

Fig. 8 Dispersion characteristics for the dominant mode on the strip waveguide, for various aspect ratios. (Figure permission acquired from Oliner (1976))

the dispersion equation (Adkins et al., 1969):

$$
\tan \left(\sqrt{k_{m R}^{2}-\beta^{2}} w\right)=\sqrt{\frac{\beta^{2}-k_{R}^{2}}{k_{m R}^{2}-\beta^{2}}}
$$

where $\beta$ is the propagation wavenumber of the guided mode, and $k_{R}$ and $k_{m R}$ are the wavenumbers of the Rayleigh wave and the modified Rayleigh wave, respectively. The corresponding phase velocities of each wavenumber are $v_{p}=\omega / \beta$, $v_{R}=\omega / k_{R}$ and $v_{m R}=\omega / k_{m R}$. In addition, Freund (1971) adopted an alternative method to obtain the dispersion relations for the waveguides, in which one side of the guiding structure was considered to be infinite so that there was only one discontinuity in the boundary conditions. Solving the three-dimensional elasticity problem enabled the investigation of the reflection and transmission of surface waves at this discontinuity. The three unknown and nonzero displacement vectors could be solved by either Laplace transform methods or the Wiener-Hopf technique (Freund et al., 1968). The results could thus be applied to geometrical characterization of dispersion relations for the propagation of the unattenuated guided surface waves.

So far the discussion has only included straight waveguides. Overcoming the anisotropy of the substrate in order to more completely control the SAW propagation is a key purpose of waveguides, and so the effects of turning waveguides is important. According to Tiersten et al. (1973), the planar scalar wave approximation procedure with large radius-toguide-width ratios $a / b$ (see Fig. 9) were extensively studied. They obtained the dispersion curves by solving Bessel's differential equations employing approximate bound modes in the partially bound wave functions and concluded that a critical $a / b$ ratio exists which depended upon the deposited and substrate materials. Beyond this critical $a / b$ ratio, the waveguide would fail to retain the wave and energy would be lost from it.

In the work cited so far, the substrate was assumed to be isotropic to facilitate analysis, but this precludes piezoelectricity and therefore the generation of the SAW in the substrate. However, in the specific case of single-crystal LN, modifications necessary in the analysis due to the anisotropy have been carried out by Schmidt et al. (1975). Based on a few simplifying assumptions about the propagation of SAWs, they analyzed the dispersion relation by assuming a single scalar potential in the wave equation. They considered the wave, which propagated along the guiding layer, in terms of modified Rayleigh wave rays that were completely reflected from the side walls of the strip. Since in anisotropic substrates such as LN that the velocity of Rayleigh waves symmetrically vary about the waveguide axis, the propagation wavenumber of the guided mode $\beta^{\prime}$, corresponding to the propagating angle $\xi$ with respect to the guide axis, may be simply corrected by using

$$
\beta^{\prime}=k_{m R}(\xi, t) \cos \xi
$$




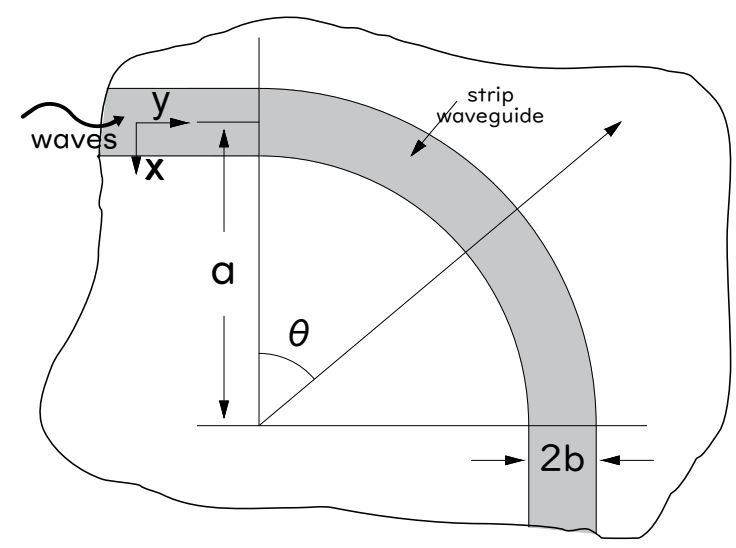

Fig. 9 Circular guide connecting two straight guides normal to each other. (Figure redrawn from Tiersten et al. (1973))

where $k_{m R}(\xi, t)$ is the wavenumber of the modified Rayleigh wave along the $\xi$ direction in the region of the deposited thin film with thickness $t$. The waves were assumed to propagate in a zigzagging path with the reflection angle $\xi$, as shown in Fig. 6. The transverse decay constants may be expressed as

$$
k_{m R}^{\prime}=\sqrt{k_{m R}^{2}(\xi, t)-\beta^{\prime 2}}
$$

for the slow region and

$$
k_{R}^{\prime}=\sqrt{\beta^{\prime 2}-k_{R}^{2}}
$$

for the fast region. The dispersion relation may thus be modified and written as

$$
\begin{aligned}
& \frac{1}{2} w \sqrt{k_{m R}^{2}(\xi, t)-\beta^{\prime 2}}=i \frac{\pi}{2}+\tan ^{-1} \sqrt{\frac{\beta^{\prime 2}-k_{R}^{2}}{k_{m R}^{2}(\xi, t)-\beta^{\prime 2}}}, \\
& \frac{1}{2} w \sqrt{k_{m R}^{\prime 2}(\xi, t)-\beta^{\prime 2}}=i \frac{\pi}{2}+\tan ^{-1} \sqrt{\frac{\beta^{\prime 2}-k_{R}^{\prime 2}}{k_{m R}^{\prime 2}(\xi, t)-\beta^{\prime 2}}}
\end{aligned}
$$

in which $i$ indexes the propagation mode. Later they further applied a parabolic approximation (Szabo et al., 1973) and finally expressed the wavenumber as

$$
\begin{aligned}
& k_{m R}(\xi)=k_{m R}(\xi=0)\left(1+\alpha \xi^{2}\right) \\
& k_{m R}^{\prime}(\xi)=k_{R}^{\prime}(\xi=0)\left(1+\alpha \xi^{2}\right)
\end{aligned}
$$

where $\alpha$ is the parabolic anisotropy factor of the substrate. The value of $k_{m R}^{\prime}$ from Eq. (8) may then be substituted into Eq. (1) wherever $k_{m R}$ appears to approximate the effects of the anisotropic media. As they concluded, this modification was valid for small angles of deviation from the guided axis, as typically found in high aspect ratio thin films.

\subsubsection{Shorting-strip waveguides}

The shorting-strip waveguide was first described by Engan (1969). The structure includes a very thin strip of metal on a piezoelectric substrate. Rather than a mass loading effect from the strip as used in strip waveguides, here the electric field associated with the piezoelectric surface wave will be short circuited by the metal strip, permitting free flow of current as the SAW passes through, reducing the net stiffness of the material and likewise the wave propagation velocity in the substrate under the strip. Unlike strip waveguides, the metal can be much thinner as all that is required is the ability to carry sufficient current to maintain the short-circuit condition. Due to how it reduces the velocity via the short circuit, this kind of waveguide is also called a $\Delta v / v$ waveguide, an unfortunate definition since all waveguides operate on the $\Delta v / v$ effect. The dispersion relation is similar to Eq. (1), but the approximate correction described in Eq. (8) is necessary because the mechanism of wave confinement under the shorting strip requires piezoelectric coupling that only 


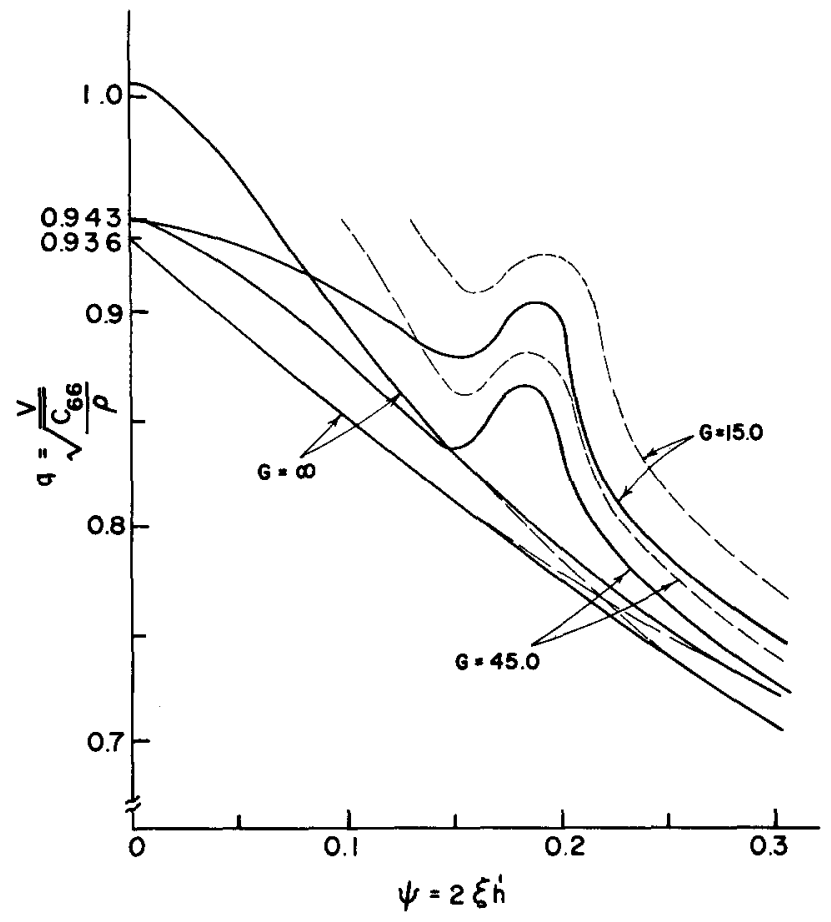

Fig. 10 Dimensionless phase velocity dispersion curves for essentially Rayleigh-type surface waves guided by strips of gold plated on a $Y$-cut LN substrate for $X$-propagation for various width-to-thickness ratios. The solid curves represent the lowest symmetric mode and the dotted, the lowest antisymmetric mode with respect to the width of the plating. (Figure permission acquired from Sinha et al. (1973))

appears in an anisotropic substrate. The reduction of velocity $(\Delta v)$ produced by short circuiting the substrate under the strip is typically small, so the guiding action provided by the strip is weak. However, as the guided wave propagates, the transverse width of most shorting-strip waveguides is constant and if the wave is able to be initially confined it should remain confined.

Hughes (1972) simply assumed the open-to-short circuit wave velocity difference $\Delta v$ to be the dominant factor in guiding SAW within the shorting strip. His theoretical work-adopting an isotropic mode which incorporated the velocity difference-adequately predicted the major features of this kind of guiding structure: strong guidance and low dispersion for many common piezoelectric materials (e.g., LN). However, failing to take the effects of the film stiffness and inertia into account, his results did not agree well with experimental results (Coldren et al., 1973), as he used wavenumbers along the guide axis rather than the actual SAW path $\xi$ that zigzagged along and back and forth across the shorting strip. Sinha et al. (1973) later included the influence of the stiffness, inertia, and electrical shorting of the deposited film and worked out the depth dependence of the full straight-crested surface wave solution from the three-dimensional equations of linear piezoelectricity (Tiersten, 2013), so that the associated dispersion relation could also be obtained. One of the results they achieved involved the dispersion curves, with different values of aspect ratio $G$, for guided SAWs propagating in both $x$ and $z$ directions on $Y$-cut LN, where thin gold films were deposited. It revealed that the dispersion curve possessed an unusual hump for the $X$-propagating SAW on LN (see Fig. 10), indicating two ranges of nondispersive propagation. They further attributed this to the strong mode coupling of the two straight-crested surface waves in the propagating direction, the extended Rayleigh wave and the fundamental quasi-Love wave. They approximated the wave propagation with a single scalar surface wave function, which described only one mode, precluding the application of the analysis to the fully mode coupled case.

The relative velocity reduction for an aluminum strip on YZ LN, with respect to the shorted surface wavenumber, was reported by Schmidt et al. (1975) (see Fig. 11), where $v_{p}$ and $v_{g}$ denoted phase velocity and group velocity, respectively, and $\alpha$ stood for anisotropy factor of the substrate. The dimensionless velocity reduction could be very small, about one to two percent. The substrate anisotropy obviously made a nontrivial contribution to the dispersion of the waveguide, if one compared the solid and dashed lines. Their measured data— the black dots and triangles—sufficiently supported the calculated relation. Furthermore, as the anisotropy factor $\alpha=0.25$ closely approximated the shorted velocity of LN, the zigzagging ray direction $\xi$ was computed to be about $7^{\circ}$, which suggested that the SAW would be confined in the "slow" 


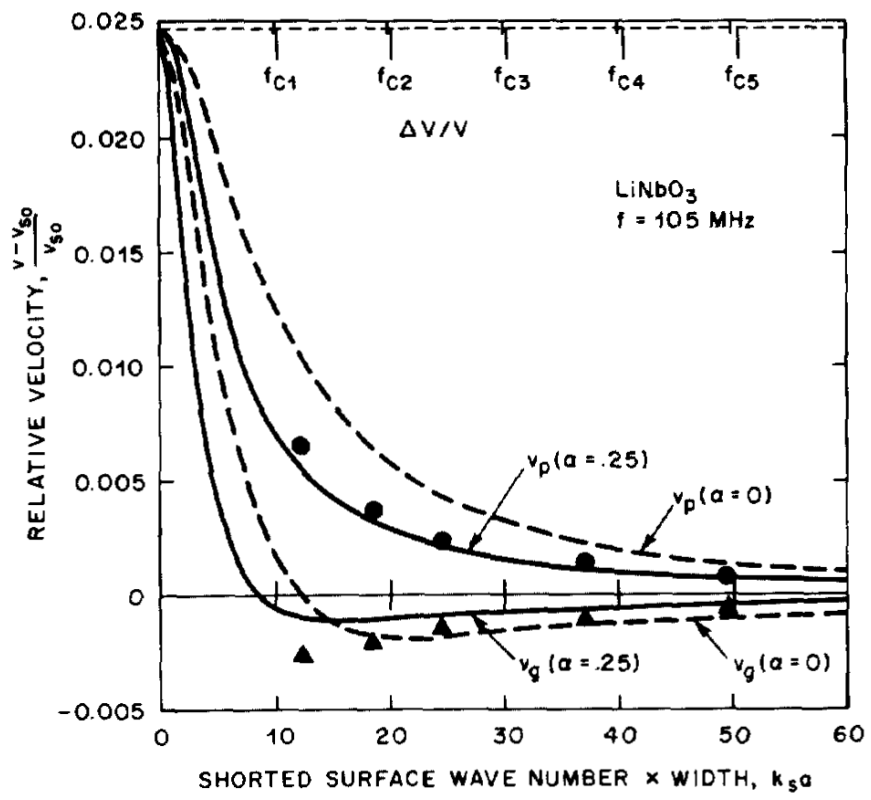

Fig. 11 Theoretical dispersion curves of $\Delta v / v$ waveguide on $\mathrm{LiNbO}_{3}$, with anisotropy included $(\alpha=0.25)$ and without anisotropy $(\alpha=0)$; phase velocity (black dots), group velocity (black triangles). Measurements at fixed frequency for guides of varying width. (Figure permission acquired from Schmidt et al. (1975))

region and would propagate back and forth at this angle (see Fig. 6).

Since both mass-loading and piezoelectric shorting can be present at the same time, it is important to note the result in Fig. 11 omits the dispersive effects of mass loading of the $30 \mathrm{~nm}$ thick aluminum strip. Schmidt et al. (1975) considered this effect as well with an $180 \mu \mathrm{m}$ wide and $50 \mathrm{~nm}$ thick aluminum strip on a YZ LN substrate (see Fig. 12), in which $v_{s}$, $v_{p}, v_{g}, \alpha$ and $\delta_{s}$ represent the wave velocity in the slow region, the phase velocity, the group velocity, the anisotropy factor, and the mass loading coefficient, respectively. When the mass loading effect was taken into account, the solid curves for phase velocity and group velocity were plotted using the approximation method mentioned above, which had a downward sloping curve, rather different than when only the shorting effect of the guiding strip (dashed lines) was considered. From their finding, it is reasonable to omit the mass loading effect on the wave propagation when the metal film is very thin and the SAW is at a relatively low frequency. But most importantly, they showed that the positive dispersion curve from the short-circuiting behavior of the shorting strip waveguide could be balanced with the mass loading of the metal in the waveguide to eliminate the dispersion—in their case, from $45 \mathrm{MHz}$ to about $120 \mathrm{MHz}$.

\subsection{Topographic waveguides}

Absent using a second material, surface waveguiding can be realized by a sufficient localized geometric shaping of the substrate surface, which is distinct from the overlay waveguiding structure discussed in the previous subsection. Rather than seek to guide waves by controlling the spatial distribution of the wave speed, the waves are instead guided by reducing the restraining forces acting on the substrate through the presence of local geometry-a ridge of material, for example-and entrapping the wave into the local geometry. This form of waveguide tends to bound the wave rather well, reducing loss to the surrounding structure in comparison to other waveguide geometries. These waveguides furthermore have no real analogy in electromagnetics. There are two important topographic waveguide shapes: ridge and wedge structures. The two typical but distinct wave propagation modes in such waveguides will next be considered.

\subsubsection{Antisymmetric flexural mode}

A typical ridge waveguide has a width $W$ and height $H$ and is bound at its base to the substrate and is free along the top edge as shown in Fig. 5(d). When $W$ is sufficiently small, it is convenient to treat the fields in the ridge region as different plate modes of propagation. In the antisymmetric flexural (ASF) ridge mode—-the odd mode—particle displacement transverse to the long axis of the ridge and the wave propagation direction is significant. The low stiffness of the ridge in this type of lateral motion causes the velocity of waves formed from it to be well below the Rayleigh velocity. The term "flexural" indicates the motion in the ridge region rocks back and forth as the wave travels down it (see Fig. 15 (a-c) and Fig. 16(a)). Mason et al. (1971) have shown the dispersion results for the ASF in the form of a normalized phase velocity 


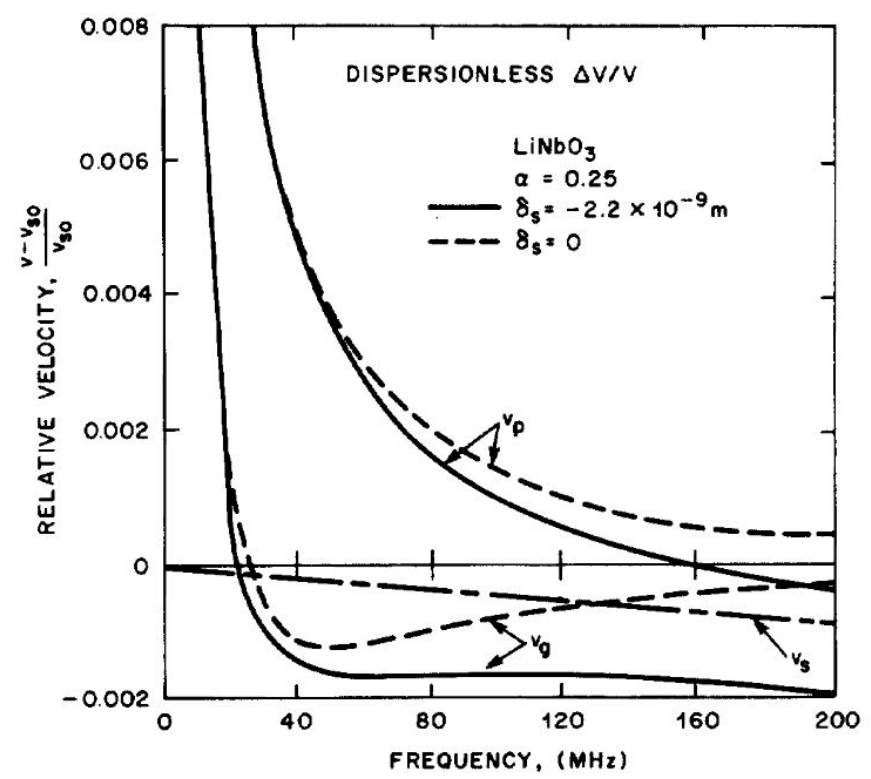

Fig. 12 Dispersion curves for the aluminum shorting-strip waveguide, taking into account the mass loading and short-circuit effects of the metal waveguide material, showing group velocity dispersion compensation. Dashed curves represent the shorting effect only. Solid curves include slight mass loading dispersion compensation (represented by the $v_{s}$ curve). (Figure permission acquired from Schmidt et al. (1975))

as a function of $\left(\omega W / v_{R}\right)$ with their experimental data. As shown in Fig. 13, the lowest curve is the analytical solution to the lowest ASF mode for an infinite plate (i.e., $H / W \rightarrow \infty$ ) (Meeker et al., 1964). Comparing the experimental results for ridge guides of different $H / W$ values, it is reasonable to conclude that, at high frequencies, the ASF phase velocity asymptotically converges to the velocity of the Lamb plate mode (lowest ASF mode) (Mason et al., 1971); while at low frequencies, the phase velocity increases rapidly towards infinity as a low-frequency cutoff. An infinite phase velocity corresponding to a finite frequency apparently indicates an infinite guiding wavelength, therefore the ridge must vibrate monolithically along its entire length as a cantilever at this cutoff. In this cantilever resonance, the energy leaks from the guide to a bulk mode that freely propagates underneath. Experiments have revealed that, in the range $0.75<H / W<3$, the rectangular ridge guide is estimated to be one quarter of an ASF Lamb mode high.

In a less rigorous approximation, it is reasonable to regard the ASF modes as traveling and non-destructive interference patterns of the fundamental mode that is specularly reflected by the impedance discontinuities at the ends of the ridge guide. With this in mind, the phase condition

$$
2 k_{s} H \cos \theta-\phi_{1}-\phi_{2}=2 m \pi
$$

must be satisfied, where $k_{s}$ is the wavenumber of substrate, and $\phi_{1}$ and $\phi_{2}$ are the phase changes of the ASF waves at the free (top) end of the ridge and at the ridge's base at the substrate, respectively. The phase changes can be constrained as $\pi$ or 0 upon the assumption that the discontinuities are equivalent to open- and short-circuits. As a result, the terms $\phi_{1}$ and $\phi_{2}$ can be simplified and produce a mode number change from $2 m$ to $2 m-1$. Introducing the wavenumber of guide $k_{g}=k_{s} \sin \theta$, the dispersion relation can be derived by integrating the ridge mode product of phase and group velocities and applying the quarter-wavelength cutoff condition:

$$
k_{g}^{2} \approx k_{s}^{2}-\left(\frac{n \pi}{2 H}\right)^{2}
$$

where $n$ is an odd integer denoting the order of the ASF mode. Dispersion characteristics for the ASF ridge mode can be plotted with Eq. (10). The calculated results were compared with the experimental data by Lagasse et al. (1972). This simple model predicted the dispersion characteristics quite well for tall ridge structures $(H / W \geq 3)$. The error was reported to be only $3 \%$ for $H / W=3$. However, when the guide is shrunk to $H / W=1.0$, the discrepancy increases to $15 \%$, which can be explained as the energy propagating in the substrate becomes more significant, according to this theory.

Later, in the work of Oliner et al. (1971) and Li et al. (1972b), a more accurate transverse resonance formulation was established by treating the substrate to be coupled to shear-horizontal ( $\mathrm{SH}$ ) bulk waves instead of simply a free surface. 
This approach produced a corrected dispersion relationship of

$$
\left(k_{s}^{2}-k_{g}^{2}\right)^{1 / 2}=\left(\frac{k_{s}}{k_{g}}\right)^{2}\left(k_{s}^{2}-k_{b}^{2}\right)^{1 / 2} \cot \left[\left(k_{s}^{2}-k_{g}^{2}\right)^{1 / 2} H\right],
$$

where $k_{b}$ was the bulk shear wavenumber. Comparing this new result with experiments (see Fig. 14) shows a substantial improvement over the past models.

Lagasse et al. (1972) has also proposed to apply finite-element analysis in determining the characteristics of the ridge mode waveguides, by dividing the cross section of the guiding structure into sufficiently small—usually triangular (Silvester, 1969; Fried, 1971)—elements. Both the anticipated gradients of the field quantities and the geometry of the guide determine the number of elements. These elements can then be defined by simple polynomial shape functions to produce a linear algebraic representation for the complete system with-fortunately-linear solutions at first order (Burridge et al., 1971). A matrix eigenvalue equation is generated with application of the finite-element method to the variational principle-derived representation,

$$
[S][U]=\omega_{0}^{2}[T][U],
$$

where $[S],[U]$ and $[T]$ are the stiffness tensor, field displacement tensor, and mass tensor, respectively, and $\omega_{0}$ is the mode eigenfrequency. By applying appropriate boundary and symmetry conditions, Fig. 15(a-c) depicts the three ASF particle deformation patterns of a tall ridge guide, in which the particle motion is normal to the direction of propagation. When it comes to the case of piezoelectric substrates, one interesting fact worth noting is that considering piezoelectricity will not reduce the dimensionality of the linear algebra representation of the system. Armstrong et al., in a private communication, had shown that the finite-element method worked accurately in obtaining an estimation of $\Delta v / v$, even for a materials with weak piezoelectricity. Their calculation of the CdS and lead zirconate titanate (PZT)-4A confirmed this accuracy, producing only 0.2 percent error in a comparison with the exact analytical value of $\Delta v / v$.

\subsubsection{Symmetric pseudo-Rayleigh mode}

Unlike the ASF mode, which has strong dispersion, the symmetric mode is almost dispersionless over a very wide frequency range, especially for ridges with high aspect ratio. The acoustic field in the waveguide distinctly resembles a Rayleigh wave, and that is why it is also named the pseudo-Rayleigh mode. As shown in Fig. 15(d) and Fig. 16(b), the constituent waves displace vertically in the tall ridge rather than horizontally.

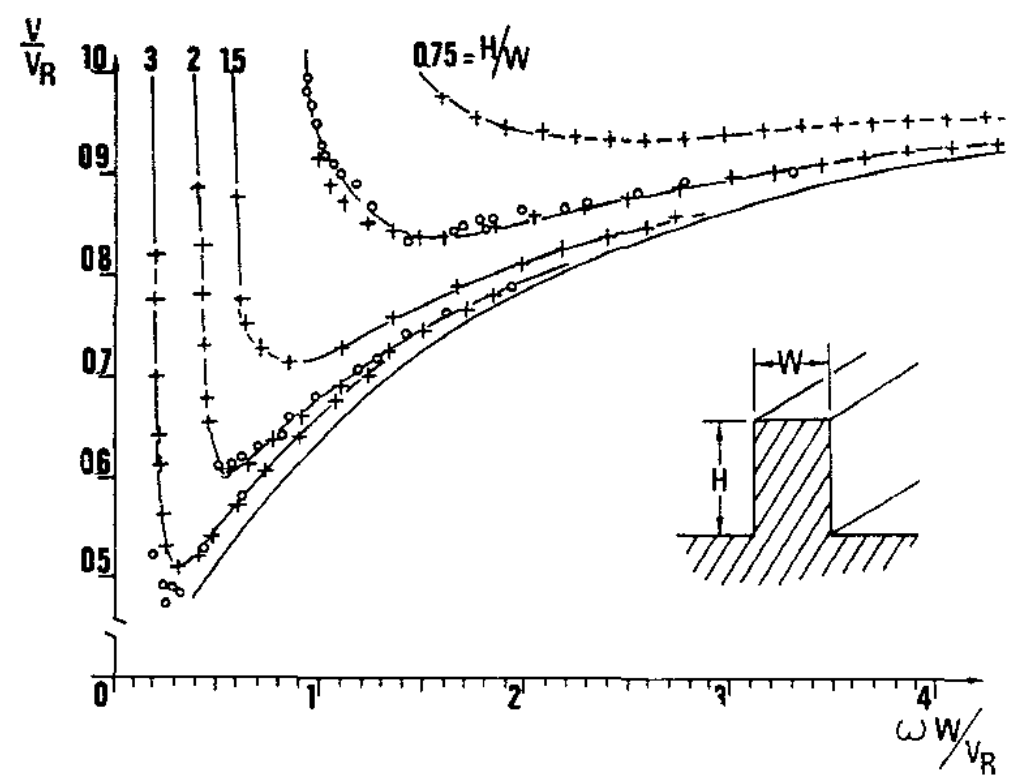

Fig. 13 The rectangular ridge waveguide (inset), with measured dispersion characteristics of the fundamental ASF mode. The parameter is $H / W$. Circles denote laser probe results, crosses the results of ring resonator experiments. The dispersion characteristics of the fundamental ASF Lamb wave is also shown. (Figure permission acquired from Lagasse et al. (1973)) 


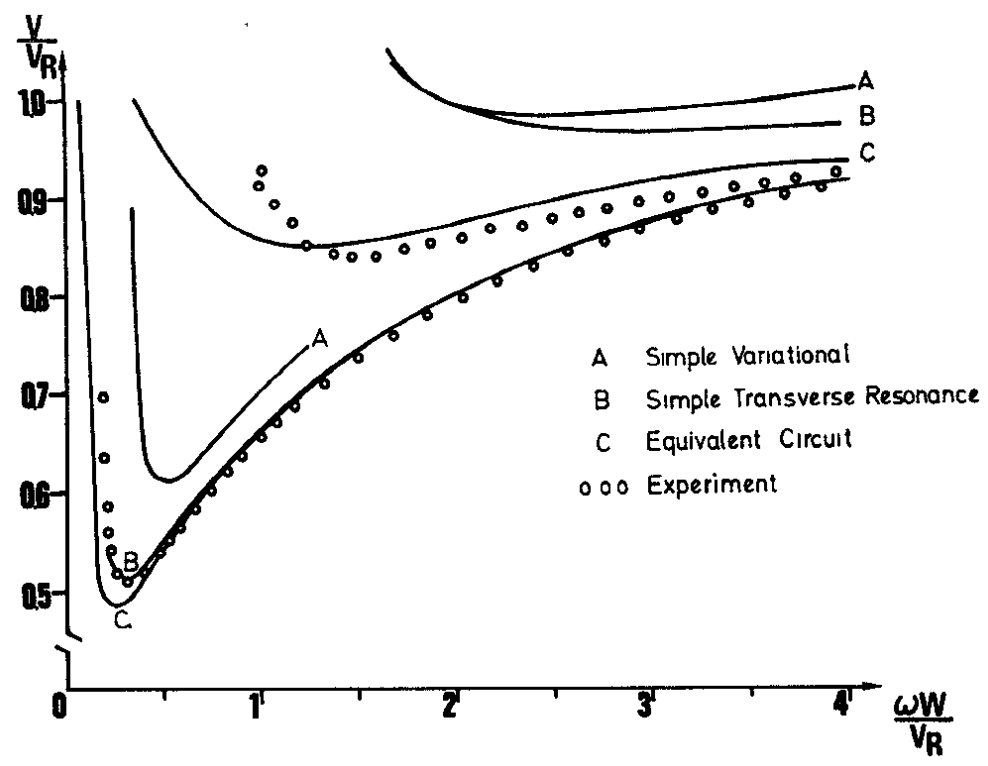

Fig. 14 Fundamental ASF mode approximation theories compared with experiment for tall $(H / W=3)$ and short $(H / W=1)$ duralumin guides. The upper right curves are for tall ridges and the lower left curves are for short ridges. Curve A: Simple variational model. Curve B: Simple transverse resonance model. Curve C: Equivalent circuit model. Dots are the experimental data. (Figure permission acquired from Lagasse et al. (1973))

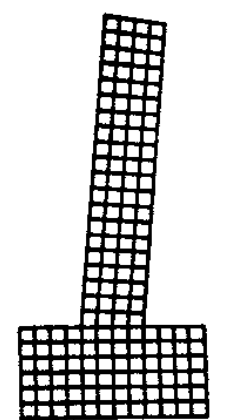

(a)

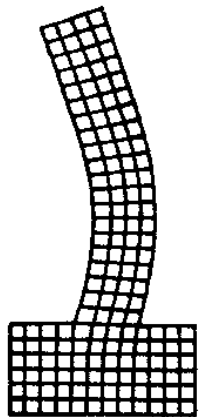

(b)

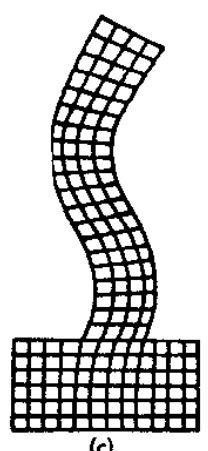

(c)

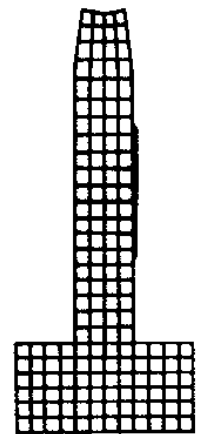

(d)

Fig. 15 Displacement patterns of four tightly bound modes that exist in a high aspect ratio $(H / W=5)$ rectangular single-material ridge waveguide. (a)(b)(c) ASF mode, (d) symmetric mode. (Figure permission acquired from Lagasse et al. (1973))

In this mode, the lowest symmetric Lamb mode and the lowest SH mode couple together at the top of the guide so that the $p$ and $s$ bulk waves combine to form a Rayleigh wave at a free planar surface. The dispersion relation was investigated by Markman et al. (1977) and Li et al. (1972a), using a transverse equivalent network approximation

$$
\left(k_{s h}^{2}-2 k_{g}^{2}\right)^{2} \frac{\sqrt{k_{g}^{2}-k_{l}^{2}}+\sqrt{k_{g}^{2}-k_{c}^{2}} \tanh \sqrt{k_{g}^{2}-k_{l}^{2}} H}{\sqrt{k_{g}^{2}-k_{c}^{2}}+\sqrt{k_{g}^{2}-k_{l}^{2}} \tanh \sqrt{k_{g}^{2}-k_{l}^{2}} H}=4 k_{g}^{2} \sqrt{k_{g}^{2}-k_{s h}^{2}} \sqrt{k_{g}^{2}-k_{l}^{2}},
$$

where $k_{s h}, k_{c}$ and $k_{l}$ were the shear, compressional wave, and lowest symmetric Lamb wave wavenumbers, respectively. Experimental data is also shown in Fig. 17, using values from Yen et al. (1975). It has been pointed out (Oliner, 1976) that the dispersion relation in Eq. (13) can only be valid for frequencies below $k_{R} W=2$ ( $k_{R}$ is the Rayleigh wavenumber). Additional transverse propagation modes would need to be considered when attempting to determine the motions in the waveguide above this frequency, and Eq. (13) would need to be adjusted accordingly.

It is known that at zero frequency, all energy is kept in the substrate and the dispersion curve infinitely approaches the Rayleigh wave velocity. At very low frequency, the effect of the substrate exists as the field decay is still small. When frequency is above a certain threshold, which seems to be rather low, the particle motion associated with the wave is mostly confined within the guide, similar to symmetric waveguide modes propagating along the ridge. Over a very wide 
frequency range, the symmetric pseudo-Rayleigh mode in the waveguide has almost no dispersion, thus making it useful in long delay line applications and potentially in acoustofluidics.

\subsubsection{Wedge}

In 1972, Lagasse et al. (1972) further studied the fundamental ASF modes of the dispersion characteristics in two trapezoidal ridge guides (see Fig. 18): a truncated knife edge and a dovetail ridge, in which $H=W$. The dispersion characteristic becomes flattened as the top of the guide narrows. The waveguides can become nearly dispersion-free if the slope angle keeps increasing and eventually forms a knife edge. According to analysis (Lagasse, 1972), the dispersion curve for an ideal wedge (substrate is infinitely far away) is a straight line; i.e., the velocity does not change with respect to frequency. This can be understood as such tall knife-edge structures have no characteristic dimension. For a more realistic case - where the height of the wedge is not infinitely large - a portion of the energy dissipates into the substrate and the wave velocity increases at low frequencies. At greater frequencies, the wave velocity stays constant and the energy is essentially confined in the guiding structure, as the structure acts as an infinite wedge.

Although it was difficult to obtain an analytical solution for the wedge guides, Lagasse et al. had applied the finiteelement method, as discussed in subsection 2.4.1, towards the wedge performance in the 1970s. Their efforts in calculating the dispersion characteristics involved various conditions: for higher modes (Lagasse, 1973b; Lagasse, 1972), for different top angles $\theta$ (Lagasse et al., 1973; Lagasse, 1972), for anisotropic substrates (Lagasse, 1973b) and for the effects of a truncated or rounded wedge shape (Lagasse et al., 1974).

Rendering one of their results, though more than one mode can take place simultaneously depending on the wedge angle, Fig. 19 presents the particle displacement for the lowest order mode. One can easily tell that the particle motion is predominantly transverse to the propagation direction and this mode is tightly bounded to the tip of the wedge. A good numerical approximation of the displacement decay regarding the depth from the apex is presented in Fig. 20, where $\left|u_{1}\right|$ is the displacement amplitude and $x_{2}$ is the depth. One typical result states the amplitude drops to $10 \%$ of the maximum amplitude at depth $x_{2}=0.9 \lambda_{R} \tan \theta / 2$, where $\lambda_{R}$ is the Rayleigh wavelength. And the penetration depth of the mode will rapidly decrease as the top angle gets smaller. Further investigation has pushed the deformation amplitude decay rate to about $\exp \left(-2 x_{2} / \lambda_{R}\right)$.

Besides the finite-element calculation, the dispersion relation was also empirically solved (Lagasse et al., 1973). The guided velocity was found to have a strong dependence upon the apex angle $\theta$,

$$
v \approx v_{R} \sin (m \theta), m \theta<90^{\circ},
$$

where $v_{R}$ was the half-space Rayleigh wave velocity and $m$ was an integer denoting the mode order. The equation indicates that an potentially dispersion free mode can be achieved for small apex angles. In a $15^{\circ}$ wedge, for instance, a guided wave with velocity only quarter of $v_{R}$ can be supported. Mason et al. (1972) has concluded, and verified by experiments, the advantages of narrow-angle wedge waveguides, high acoustic field density, very low dispersion, and low velocity made them a perfect candidate for nonlinear interactions devices.

Although the acoustic waves propagating at the tip of an ideal infinite wedge waveguide is dispersionless, the weak

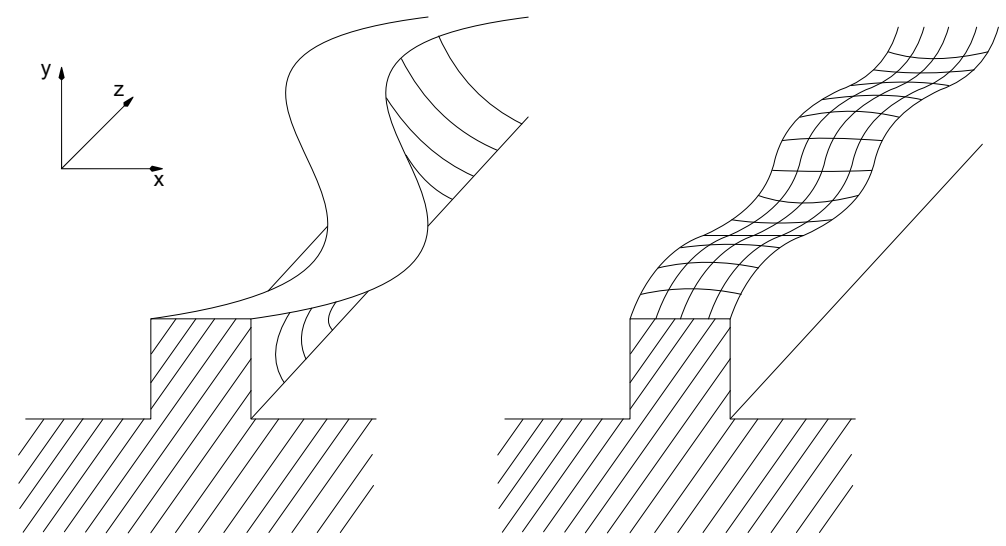

(a)

(b)

Fig. 16 Sketch of propagation modes in the ridge guides. (a) ASF mode (particle displacement predominantly in $x$ and $z$ directions), (b) pseudo-Rayleigh mode (particle displacement predominantly in $y$ and $z$ directions). (Replotted using the data from Mason et al. (1971)) 


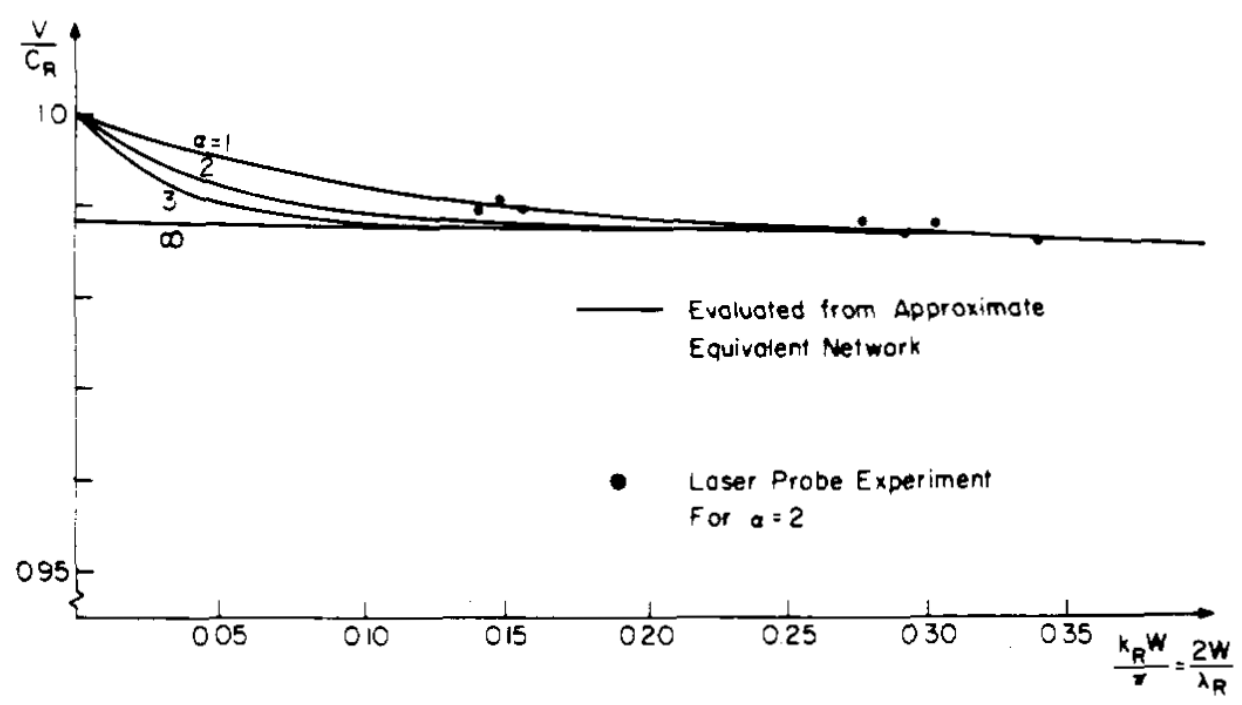

Fig. 17 Dispersion characteristics for the dominant symmetric pseudo-Rayleigh mode on the topographic rectangular ridge waveguide, using an expanded ordinate scale to show the effect at low frequencies of different aspect ratios. (Figure permission acquired from Oliner (1976))

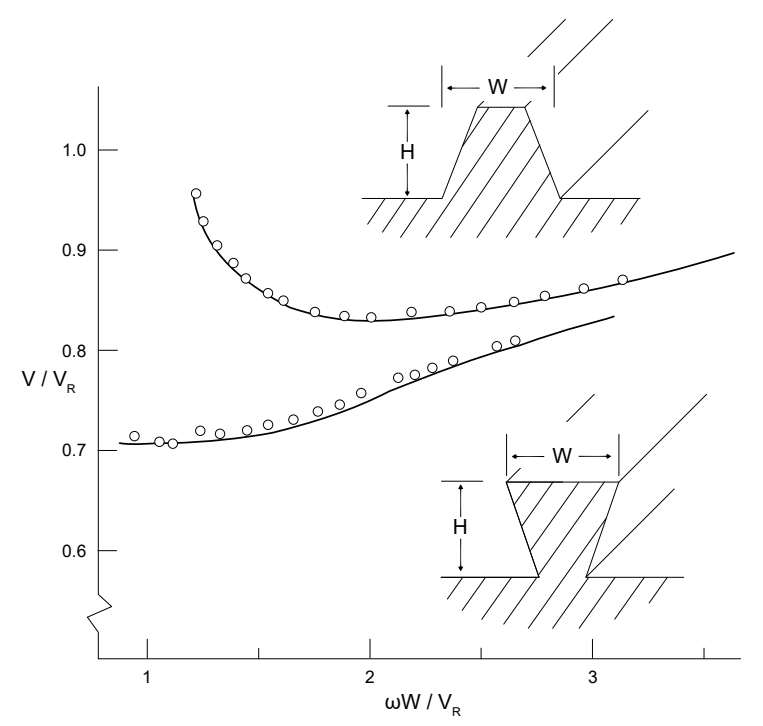

Fig. 18 Theory compared with experiment for the fundamental ASF mode in trapezoidal duralumin ridges. $H / W=1$. Sides deviate from substrate normal by $15^{\circ}$. (Replotted using the data from Lagasse et al. (1972))

dispersion still attributes to a number of factors. As a result, in later years, wedge waves can be used in non-destructive testing (Wang et al., 2007; Liu et al., 2010), as modifying the geometry of the wedge can apparently change the dispersion relation. And sometimes the dispersion is intentionally introduced to produce nonlinear effects as needed (Krylov et al., 1992; Krylov et al., 1993; Mayer et al., 1997; Mayer et al., 2009).

Sokolova et al. (2013) applied perturbation theory on the early work of Lagasse et al. (1974) to investigate the dependence of frequency or phase velocity on the wavenumber (inverse wavelength). They found that when one or two surfaces of the wedge were coated by a thin film (see Fig. 21(a)), the dispersion law turned to be

$$
\omega^{2}=\left(v_{p} k\right)^{2}\left(1-2 k d \times F_{1}\right)
$$

where $\omega, v_{p}$ and $k$ were the angular frequency, phase velocity, and wavenumber, respectively, and $d$ was the thickness of the thin film coating. The dimensionless constant $F_{1}$ depended on the acoustic mismatch of the two materials, such as density and elastic modulus, and the angle of the wedge. Besides, when a small part of the cross section of the wedge close to the tip was changed, for example, truncation (see Fig. 21(b)), rounding of the tip, or replacement by another material, the dispersion relation became

$$
\omega^{2}=\left(v_{p} k\right)^{2}\left(1-2 k A_{1} \times F_{2}\right)
$$




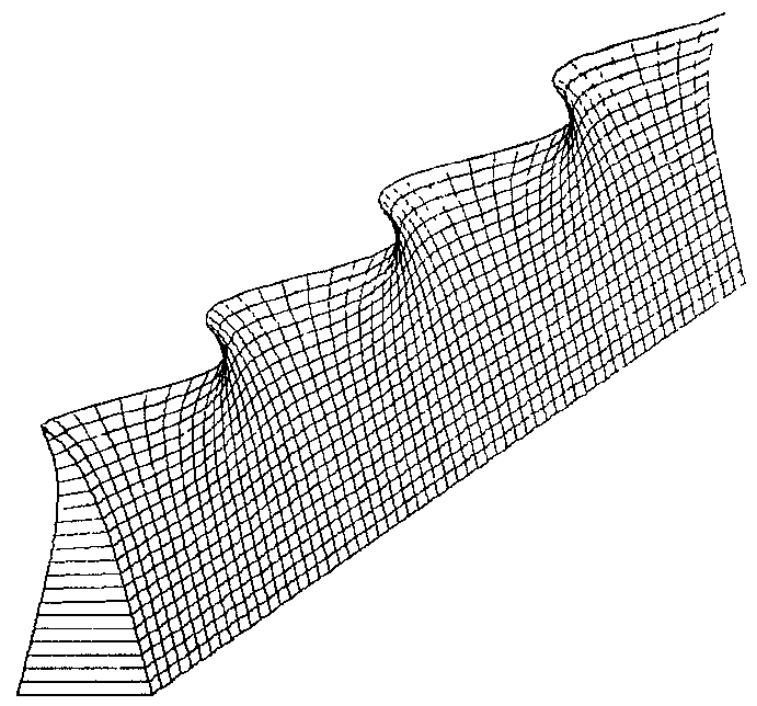

Fig. 19 Computer-generated perspective view of propagation in the lowest order mode on a narrow apex wedge. Wave motion is predominantly antisymmetric flexural. (Figure permission acquired from Lagasse et al. (1973))

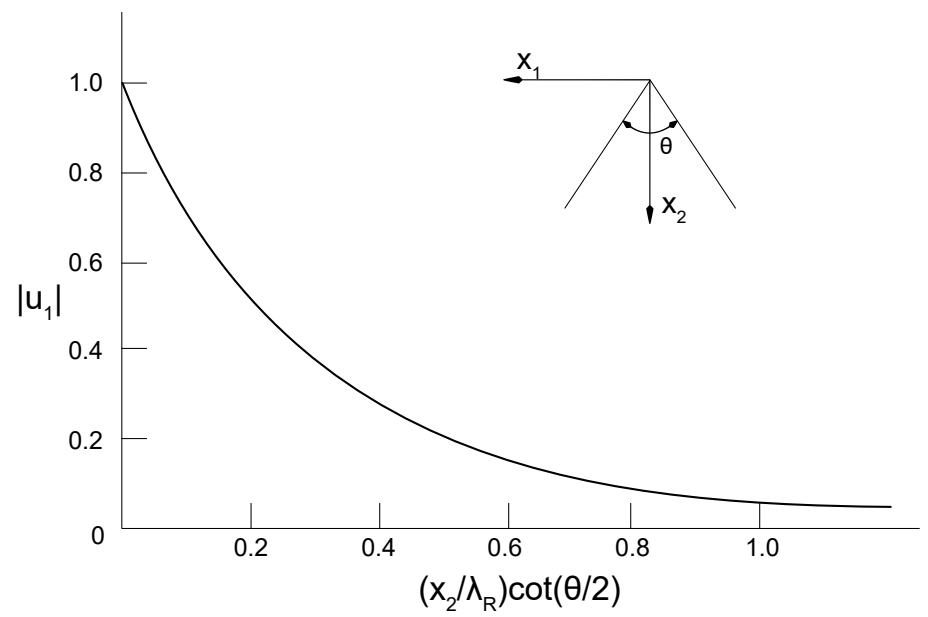

Fig. 20 Displacement amplitude as a function of depth. (Replotted using the data from Lagasse et al. (1972))

where $A_{1}$ was the cross-section that was removed or replaced, and coefficient $F_{2}$ depended upon the acoustic mismatch between the filling material and the wedge. The finite element analysis (FEA) simulation of Hladky-Hennion (1996) confirmed that the modification of the tip strongly induced a dispersion of wedge wave velocity at small angles.

In more recent years, research has analyzed a type of highly localized plasmon — channel plasmon polaritons (CPP)— -in metallic grooves, which showed superior features in subwavelength guiding with strong localization and low dispersion (Bozhevolnyi et al., 2006; Gramotnev et al., 2004; Pile et al., 2004). The structure could be regarded as a reverse of the wedge waveguide, where a "V" shape was cut into the substrate. In the context of acoustic waves, Liu et al. (2009) simulated the dispersion relation by the compact two-dimensional finite-difference time-domain method, and concluded that when the V-groove was immersed in water, most of the elastic energy would be confined in the fluid domain at the bottom. They also calculated the cutoff angle of the groove, $90^{\circ}$, specifically for poly(methyl methacrylate) (PMMA), otherwise the groove waves would leak into the two flat surfaces. This new type of waveguide could also be promising in piezoelectric materials.

\subsection{Direct change of surface property on lithium niobate}

The biggest problem with overlaid—shorting and mass-loading — waveguides is the fact that a layered substrate is lossier than an unlayered one. As a result, apart from the mass-loading and shorting-strip waveguiding structures, there is another route to waveguide structure fabrication that adds negligible loss but still allows the SAW velocity to be 


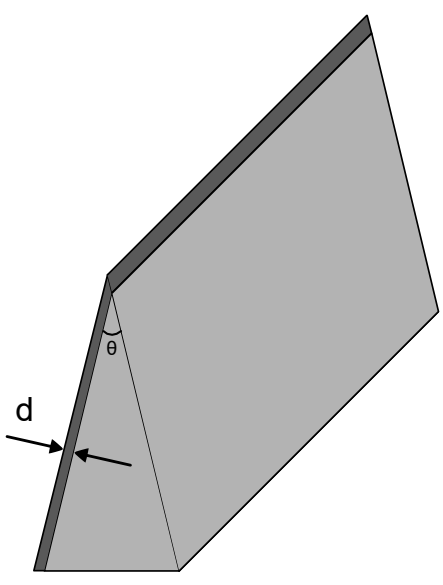

(a)

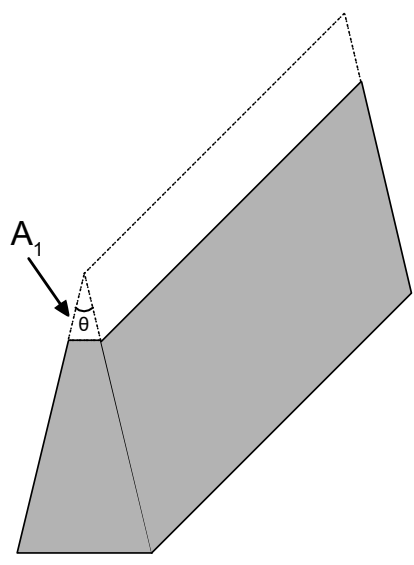

(b)

Fig. 21 Influence of wedge wave dispersion by (a) coating of one of the surfaces, (b) truncation of the tip of the wedge. (Figure redrawn from Sokolova et al. (2013))

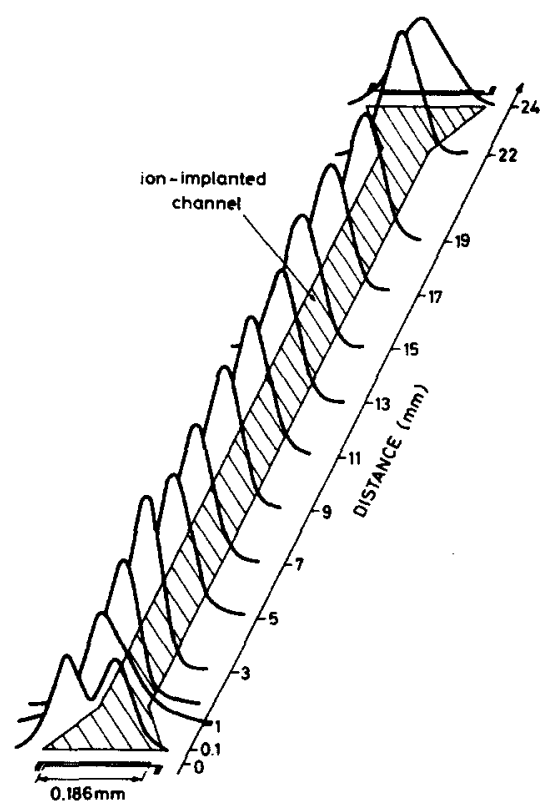

Fig. 22 Acoustic power profiles along an ion-implanted $150 \mathrm{MHz}$ SAW guide on YZ LN. The ion dose is equal to $6 \times 10^{15} \mathrm{ions} / \mathrm{cm}^{2}$ at $100 \mathrm{keV}$. The ion-implanted channel is $81.2 \mu \mathrm{m}$ wide. (Figure permission acquired from Hartemann et al. (1978))

significantly changed: directly changing the material properties of the LN itself in the substrate.

As it has long been proved that diffusion of metals or their oxides into LN could locally increase the optical refractive index of the crystal (Schmidt et al., 1974), SAW waveguide can be fabricated by creating an in-diffused region (fast region) and an non-in-diffused region (slow region). Schmidt (1975) and Weller et al. (1977) proposed that the surface wave velocity could be increased by metal in-diffusion without additional acoustic loss. A thin layer of metal (Ti, Ni, Cr) of thickness $\tau$ was evaporated onto the LN surface in order to create a region of increased velocity via metal diffusion, for which the effective depth obeyed the diffusion equation, $b=(4 D t)^{1 / 2}$, where $D$ was the diffusion constant. A typical condition could be: $\tau=100 \mathrm{~nm}, t=30 \mathrm{~h}$, and $T=1000{ }^{\circ} \mathrm{C}$. The diffused depth was reported to be $3 \mu \mathrm{m}$ when Ti was involved, resulting in an increase of velocity at about $1 \%$ for SAWs at $412 \mathrm{MHz}$. And they also pointed out that the loss for waves traveling on both diffused and undiffused region were on the order of $0.1 \mathrm{~dB}$. Their results indicated similar conclusion as that of Schmidt, and further bolstered the advantages of such waveguides: low loss, non-shorted surface, and potential dispersion control by tuning the diffusion parameters.

Other than metal diffusion, a novel means involving ion implantation, which successfully decreased the SAW velocity in LN, was proposed by Hartemann (1975) and Hartemann et al. (1978). Ion implantation was commonly utilized 


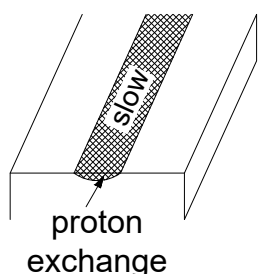

(a)

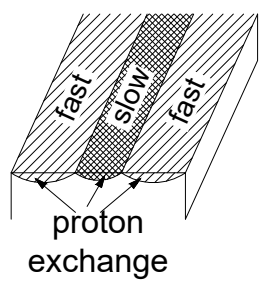

(c)

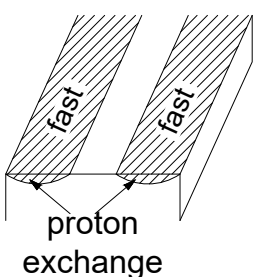

(b)

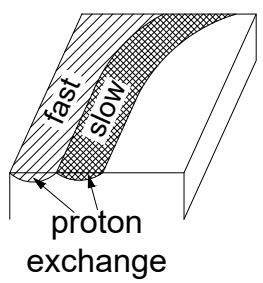

(d)

Fig. 23 Possible proton exchanged SAW waveguiding structures on YX and YZ LN (a) "stripe-type guide"; (b) "slot-type guide"; (c) combined "stripe- and slot-type" guide; (d) curved asymmetric guide. (Figure adapted from Hinkov (1987))

for fabricating semiconductor components, and the most straightforward advantage was that it did not require high temperature as metal diffused. Two doses of helium ions at $100 \mathrm{keV}$, with ion beam density at $2.5 \times 10^{15}, 6 \times 10^{15}$ and $10^{16}$ ions $/ \mathrm{cm}^{2}$, have been used in their study. As the ion bombardment would induce a velocity decrease for LN, they attributed this reduction to the change of effective electromechanical coupling coefficient. Also as shown in Fig. 22, the acoustic power distribution was investigated with a laser probe for $150 \mathrm{MHz}$ SAWs, which produced a Gaussian profile. And through their data and calculation using Knowles' scalar potential (Knowles et al., 1968), for an $81.2 \mu$ m wide ion-implanted channel, $90 \%$ of the acoustic power was confined in a region of width $120 \mu \mathrm{m}$.

In the 1980s, new ideas on producing high-index optical waveguides were devised with the exchange of $\mathrm{Li}^{+}$ion by protons in LN (Jackel et al., 1982). Proton exchange caused the density of LN to reduce, resulted from the lighter protons which substituted the heavier $\mathrm{Li}^{+}$ions and a small increase in the volume of the material from an expansion of the structure (Jackel et al., 1981). This technique led to a large decrease (up to 20\%) of phase as well as group velocity on the LN surface, because of the drastic reduction of the corresponding elastic constants. It could be used to construct acoustic waveguides (see Fig. 23) due to the strong field confinement and relative low loss (Hinkov, 1987), and superior to ion implantation as a low-cost alternative.

\section{Conclusions}

This review at first briefly described the devices and materials used to generate SAWs, later pointing out the two main problems that prevent the use of SAW on a piezoelectric substrate in microfluidics, nanofluidics, and acoustofluidics applications: beam steering and lateral diffraction. A possible solution is proposed in the form of waveguides that can not only overcome these difficulties but also confine the wave in the "slow" region and thus manipulate the direction of wave propagation on the surface. As in the context of wave confinement, devices that are able to generate relatively narrow beam SAW (FIDT) are introduced in detail. In the discussion of flat overlay waveguides, comparing with mass-loading waveguides, shorting-strip waveguides show the ability of reducing the velocity by a short-circuiting effect, producing a method for waveguiding regardless of the anisotropy of the piezoelectric substrate, especially LN. Topographic waveguides, different from thin film waveguides, are essentially single material structures with locally defined geometries. In rectangular ridge waveguides, the ASF mode shows both strong confinement and dispersion, while the pseudo-Rayleigh mode is almost dispersionless. The wedge shaped waveguide is able to efficiently confine the waves and energy at the wedge tip with minimal dispersion. Another type of guiding technique, altering the substrate's material properties, was also discussed. To sum up, thin film waveguides have been proven to possess the advantages of low loss propagation and ability to readily follow any waveguide bends, such as acoustic wave steering and splitting using different waveguide structures, which could be useful in enhancing the devices performance. Furthermore, by incorporating waveguides, it is 
promising to allow wave propagation in arbitrary direction and drive acoustic energy to independent locations to achieve fluid or particle manipulation at disparate positions, which can be widely applied in the field of acoustofluidics.

\section{Acknowledgements}

This work was in significant part generously supported by a research grant from the W.M. Keck Foundation, the National Institutes of Health Interdisciplinary Anesthesiology Research Training Program T32-GM121318-01, a grant from the Office of Naval Research (12368098), and a grant from the Department of Energy (DE-EE0008363). The authors are grateful to Prof. Kenjiro Takemura for advice and comments regarding this work.

\section{References}

Adams, P. and Shaw, H., Surface acoustic waveguide for long time delay, 1972 IEEE Ultrasonics Symposium (1972)

Adkins, L. and Hughes, A., Elastic surface waves guided by thin films: gold on fused quartz, IEEE Transactions on Microwave Theory and Techniques, Vol. 17, No. 11 (1969), pp. 904-911

Ash, E. A., De La Rue, R., and Humphryes, R., Microsound surface waveguides, IEEE Transactions on Microwave Theory and Techniques, Vol. 17, No. 11 (1969), pp. 882-892

Bausk, E., Taziev, R., and Lee, A., Synthesis of slanted and quasi-slanted SAW transducers, IEEE Transactions on Ultrasonics, Ferroelectrics, and Frequency Control, Vol. 51, No. 8 (2004), pp. 1002-1009

Bozhevolnyi, S. I., Volkov, V. S., Devaux, E., Laluet, J.-Y., and Ebbesen, T. W., Channel plasmon subwavelength waveguide components including interferometers and ring resonators, Nature, Vol. 440, No. 7083 (2006), p. 508

Budden, K., Radio caustics and cusps in the ionosphere, Proceedings of the Royal Society of London A: Mathematical, Physical and Engineering Sciences, Vol. 350, No. 1661, The Royal Society (1976), pp. 143-164

Burridge, R. and Sabina, F., Theoretical computations on ridge acoustic surface waves using the finite-element method, Electronics Letters, Vol. 7, No. 24 (1971), pp. 720-722

Campbell, C., Surface acoustic wave devices for mobile and wireless communications (1998), Academic Press

Chladni, E. F. F., Discoveries on the theory of sound (1787), Weidmanns, Erben und Reich

Coldren, L. and Schmidt, R., Acoustic surface wave $\Delta v / v$ waveguides on anisotropic substrates, Applied Physics Letters, Vol. 22, No. 10 (1973), pp. 482-483

Collins, D. J., Ma, Z., and Ai, Y., Highly localized acoustic streaming and size-selective submicrometer particle concentration using high frequency microscale focused acoustic fields, Analytical Chemistry, Vol. 88, No. 10 (2016), pp. 5513-5522

Collins, D. J., Ma, Z., Han, J., and Ai, Y., Continuous micro-vortex-based nanoparticle manipulation via focused surface acoustic waves, Lab on a Chip, Vol. 17, No. 1 (2017), pp. 91-103

Collins, D. J., Neild, A., and Ai, Y., Highly focused high-frequency travelling surface acoustic waves (SAW) for rapid single-particle sorting, Lab on a Chip, Vol. 16, No. 3 (2016), pp. 471-479

Connacher, W., Zhang, N., Huang, A., Mei, J., Zhang, S., Gopesh, T., and Friend, J., Micro/nano acoustofluidics: materials, phenomena, design, devices, and applications, Lab on a Chip (2018)

Curie, J. and Curie, P., Contractions and expansions produced by voltages in hemihedral crystals with inclined faces, Comptes Rendus, Vol. 93 (1881), pp. 1137-1140

Dentry, M. B., Yeo, L. Y., and Friend, J. R., Frequency effects on the scale and behavior of acoustic streaming, Physical Review E, Vol. 89, No. 1 (2014), p. 013203

Destgeer, G. and Sung, H. J., Recent advances in microfluidic actuation and micro-object manipulation via surface acoustic waves, Lab on a Chip, Vol. 15, No. 13 (2015), pp. 2722-2738

Devendran, C., Gunasekara, N. R., Collins, D. J., and Neild, A., Batch process particle separation using surface acoustic waves (SAW): integration of travelling and standing SAW, Royal Society of Chemistry Advances, Vol. 6, No. 7 (2016), pp. 5856-5864

Ding, X., Li, P., Lin, S.-C. S., Stratton, Z. S., Nama, N., Guo, F., Slotcavage, D., Mao, X., Shi, J., Costanzo, F., et al., Surface acoustic wave microfluidics, Lab on a Chip, Vol. 13, No. 18 (2013), pp. 3626-3649

Ding, X., Lin, S.-C. S., Lapsley, M. I., Li, S., Guo, X., Chan, C. Y., Chiang, I.-K., Wang, L., McCoy, J. P., and Huang, T. J., Standing surface acoustic wave (SSAW) based multichannel cell sorting, Lab on a Chip, Vol. 12, No. 21 (2012), pp. $4228-4231$ 
Engan, H., Experiments with Elastic Surface Waves in Piezoelectric Ceramics (1969), Electronics Research Laboratory, Norwegian Institute of Technology

Fall, D., Duquennoy, M., Ouaftouh, M., Smagin, N., Piwakowski, B., and Jenot, F., Generation of broadband surface acoustic waves using a dual temporal-spatial chirp method, The Journal of the Acoustical Society of America, Vol. 142, No. 1 (2017), EL108-EL112

Fang, S. R., Zhang, S., and Lu, Z. F., SAW focusing by circular-arc interdigital transducers on YZ-LiNbO 3 , IEEE Transactions on Ultrasonics, Ferroelectrics, and Frequency Control, Vol. 36, No. 2 (1989), pp. 178-184

Faraday, M., On the forms and states assumed by fluids in contact with vibrating elastic surfaces, Philosophical Transactions of the Royal Society London, Vol. 121, No. 319 (1831), p. 1831

Freund, L., Guided surface waves on an elastic half space, Journal of Applied Mechanics, Vol. 38, No. 4 (1971), pp. 899905

Freund, L. and Achenbach, J., Waves in a semi-infinite plate in smooth contact with a harmonically disturbed half-space, International Journal of Solids and Structures, Vol. 4, No. 6 (1968), pp. 605-621

Fried, I., Accuracy of finite element eigenproblems, Journal of Sound and Vibration, Vol. 18, No. 2 (1971), pp. 289-295

Friend, J. and Yeo, L. Y., Microscale acoustofluidics: Microfluidics driven via acoustics and ultrasonics, Reviews of Modern Physics, Vol. 83, No. 2 (2011), pp. 647-704

Gizeli, E., Bender, F., Rasmusson, A., Saha, K., Josse, F., and Cernosek, R., Sensitivity of the acoustic waveguide biosensor to protein binding as a function of the waveguide properties, Biosensors and Bioelectronics, Vol. 18, No. 11 (2003), pp. 1399-1406

Gramotnev, D. K. and Pile, D. F., Single-mode subwavelength waveguide with channel plasmon-polaritons in triangular grooves on a metal surface, Applied Physics Letters, Vol. 85, No. 26 (2004), pp. 6323-6325

Green, J. and Kino, G., SAW convolvers using focused interdigital transducers, IEEE Transactions on Sonics Ultrasonics, Vol. 30 (1983), pp. 43-50

Green, J., Kino, G., and Khuri-Yakub, B., Focused surface wave transducers on anisotropic substrates: a theory developed for the waveguided storage correlator, 1980 Ultrasonics Symposium, IEEE (1980), pp. 69-73

Guo, F., Li, P., French, J. B., Mao, Z., Zhao, H., Li, S., Nama, N., Fick, J. R., Benkovic, S. J., and Huang, T. J., Controlling cell-cell interactions using surface acoustic waves, Proceedings of the National Academy of Sciences, Vol. 112, No. 1 (2015), pp. 43-48

Hartemann, P., Acoustic-surface-wave velocity decrease produced by ion implantation in lithium niobate, Applied Physics Letters, Vol. 27, No. 5 (1975), pp. 263-265

Hartemann, P., Cauvard, P., and Desbois, D., Ion-implanted surface-acoustic-wave guides on lithium niobate, Applied Physics Letters, Vol. 32, No. 5 (1978), pp. 266-268

Haydl, W. H., Apodised surface-wave transducers, Electronics Letters, Vol. 8, No. 20 (1972), pp. 493-494

Hinkov, V., Proton exchanged waveguides for surface acoustic waves on $\mathrm{LiNbO}_{3}$, Journal of Applied Physics, Vol. 62, No. 9 (1987), pp. 3573-3578

Hladky-Hennion, A.-C., Finite element analysis of the propagation of acoustic waves in waveguides, Journal of Sound and Vibration, Vol. 194, No. 2 (1996), pp. 119-136

Hughes, A. J., Elastic surface wave guidance by $(\Delta v / v)$ effect guidance structures, Journal of Applied Physics, Vol. 43, No. 6 (1972), pp. 2569-2586

Jackel, J. L., Rice, C., and Veselka, J., Proton exchange for high-index waveguides in $\mathrm{LiNbO}_{3}$, Applied Physics Letters, Vol. 41, No. 7 (1982), pp. 607-608

Jackel, J. and Rice, C., Topotactic $\mathrm{LiNbO}_{3}$ to cubic perovskite structural transformation in $\mathrm{LiNbO}_{3}$ and $\mathrm{LiTaO}_{3}$, Ferroelectrics, Vol. 38, No. 1 (1981), pp. 801-804

Jung, J. H., Destgeer, G., Ha, B., Park, J., and Sung, H. J., On-demand droplet splitting using surface acoustic waves, Lab on a Chip, Vol. 16, No. 17 (2016), pp. 3235-3243

Kabir, K. M., Sabri, Y. M., Kandjani, A. E., Ippolito, S. J., and Bhargava, S. K., Development and comparative investigation of Ag-sensitive layer based SAW and QCM sensors for mercury sensing applications, Analyst, Vol. 141, No. 8 (2016), pp. 2463-2473

Kharusi, M. S. and Farnell, G. W., On diffraction and focusing in anisotropic crystals, Proceedings of the IEEE, Vol. 60, No. 8 (1972), pp. 945-956 
Knowles, R. R. and Opdyke, N. D., Paleomagnetic results from the Mauch Chunk Formation: A test of the origin of curvature in the folded Appalachians of Pennsylvania, Journal of Geophysical Research, Vol. 73, No. 20 (1968), pp. 6515-6526

Krylov, V. V., Mayer, A., and Parker, D., Nonlinear evolution of initially sine-like wedge acoustic waves, 1993 Proceedings IEEE Ultrasonics Symposium, IEEE (1993), pp. 765-768

Krylov, V. and Parker, D., Harmonic generation and parametric mixing in wedge acoustic waves, Wave Motion, Vol. 15, No. 2 (1992), pp. 185-200

Kundt, A., On a new kind of acoustic dust figures and on the application of the same for determining sound velocity in solid bodies and gases, Annals of Physics, Vol. 203, No. 4 (1866), pp. 497-523

Kurosawa, M., Watanabe, T., Hutami, A., and Higuchi, T., Surface Acoustic Wave Atomizer with Pumping Effect, IEEE (1995), pp. 25-30

Lagasse, P. E., Mason, I. M., and Ash, E. A., Acoustic surface waveguides-analysis and assessment, IEEE Transactions on Microwave Theory and Techniques, Vol. 21, No. 4 (1973), pp. 225-236

Lagasse, P., Analysis of a dispersionfree guide for elastic waves, Electronics Letters, Vol. 8, No. 15 (1972), pp. 372-373

Lagasse, P., Analysis of silicon ridge waveguides for acoustic surface waves, Microwave Conference, 1973. 3rd European, Vol. 2, IEEE (1973), pp. 1-4

Lagasse, P., Finite element analysis of piezoelectric elastic waveguides, IEEE Transactions on Sonics and Ultrasonics, Vol. 20, No. 4 (1973), pp. 354-359

Lagasse, P., Higher-order finite-element analysis of topographic guides supporting elastic surface waves, The Journal of the Acoustical Society of America, Vol. 53, No. 4 (1973), pp. 1116-1122

Lagasse, P., Cabus, M., and Verplanken, M., The influence of truncation on dispersion of wedge guides, 1974 Ultrasonics Symposium, IEEE (1974), pp. 121-124

Lagasse, P. and Mason, I., Analysis of propagation in ridge guides for acoustic surface waves, Electronics Letters, Vol. 8, No. 4 (1972), pp. 82-84

Lamperski, J., SAW filters with weighted tapered interdigital transducers, IEEE 1992 Ultrasonics Symposium Proceedings, Vol. 1 (1992), pp. 203-206

Langelier, S. M., Yeo, L. Y., and Friend, J., UV epoxy bonding for enhanced SAW transmission and microscale acoustofluidic integration, Lab on a Chip, Vol. 12, No. 16 (2012), pp. 2970-2976

Li, R., Bertoni, H., Oliner, A., and Markman, S., Propagation characteristics of the pseudo-Rayleigh mode of the ridge guide for acoustic surface waves, Electronics Letters, Vol. 8, No. 9 (1972), pp. 220-221

Li, R., Bertoni, H., Oliner, A., and Markman, S., Simple equivalent network for the flexural mode of the ridge guide for acoustic surface waves, Electronics Letters, Vol. 8, No. 8 (1972), pp. 211-212

Li, R., Oliner, A., and Bertoni, H., Microwave network analyses of surface acoustic waveguides. I-Flat overlay guides, IEEE Transactions on Sonics Ultrasonics, Vol. 24 (1977), pp. 66-78

Lima Jr, M. de, Hey, R., Stotz, J., and Santos, P., Acoustic manipulation of electron-hole pairs in GaAs at room temperature, Applied Physics Letters, Vol. 84, No. 14 (2004), pp. 2569-2571

Liu, F., Peng, S., Jia, H., Ke, M., and Liu, Z., Strongly localized acoustic surface waves propagating along a V-groove, Applied Physics Letters, Vol. 94, No. 2 (2009), p. 023505

Liu, I. and Yang, C., An investigation on wedge waves and the interaction with a defect using a quantitative laser ultrasound visualization system, 2010 IEEE International Ultrasonics Symposium, IEEE (2010), pp. 817-820

Markman, S., Li, R. C., Oliner, A., and Bertoni, H., Microwave network analyses of surface acoustic waveguides. IIRectangular ridge guides, IEEE Transactions on Sonics Ultrasonics, Vol. 24 (1977), pp. 79-87

Marynowski, T., Focusing transducer for SAW beamwidth compression on YZ lithium niobate, 1982 Ultrasonics Symposium, IEEE (1982), pp. 160-165

Mason, I., De La Rue, R., Schmidt, R., Ash, E., and Lagasse, P., Ridge guides for acoustic surface waves, Electronics Letters, Vol. 7, No. 14 (1971), pp. 395-397

Mason, I., Motz, M., and Chambers, J., Wedge waveguide parametric signal processing, 1972 Ultrasonics Symposium, IEEE (1972), pp. 314-315

Mayer, A. P., Garova, E. A., and Mozhaev, V. G., Nonlinear surface and wedge acoustic waves in the presence of weak dispersion, Proceedings of the Estonian Academy of Sciences, Physics and Mathematics, Vol. 46 (1997), pp. 85-93

Mayer, A. P., Lomonosov, A. M., and Hess, P., Nonlinear acoustic waves localized at crystal edges, 2009 IEEE International Ultrasonics Symposium, IEEE (2009), pp. 1088-1091 
McCoy, J. J. and Mindlin, R., Extensional waves along the edge of an elastic plate, Journal of Applied Mechanics, Vol. 30, No. 1 (1963), pp. 75-78

Meeker, T. and Meitzler, A., Guided wave propagation in elongated cylinders and plates, Physical Acoustics, Vol. 1, No. Part A (1964), pp. 111-167

Morgan, D., Surface acoustic wave filters (2007), Academic Press

Nakagawa, Y., A new SAW convolver using multichannel waveguide, IEEE 1991 Ultrasonics Symposium Proceedings, IEEE (1991), pp. 255-258

Oliner, A., Li, R., and Bertoni, H., Microwave network approach to guided acoustic surface wave structures. Tech. rep. (1971), Polytechnic Institute of Brooklyn Farmingdale New York Department of Electrophysics

Oliner, A. A., Waveguides for acoustic surface waves: A review, Proceedings of the IEEE, Vol. 64, No. 5 (1976), pp. 615627

Pile, D. F. and Gramotnev, D. K., Channel plasmon-polariton in a triangular groove on a metal surface, Optics Letters, Vol. 29, No. 10 (2004), pp. 1069-1071

Raghavan, A. and Cesnik, C. E., Review of guided-wave structural health monitoring, Shock and Vibration Digest, Vol. 39, No. 2 (2007), pp. 91-116

Rayleigh, L., On waves propagated along the plane surface of an elastic solid, Proceedings of the London Mathematical Society, Vol. 1, No. 1 (1885), pp. 4-11

Ren, L., Chen, Y., Li, P., Mao, Z., Huang, P.-H., Rufo, J., Guo, F., Wang, L., McCoy, J. P., Levine, S. J., et al., A highthroughput acoustic cell sorter, Lab on a Chip, Vol. 15, No. 19 (2015), pp. 3870-3879

Rezk, A. R., Qi, A., Friend, J. R., Li, W. H., and Yeo, L. Y., Uniform mixing in paper-based microfluidic systems using surface acoustic waves, Lab on a Chip, Vol. 12, No. 4 (2012), pp. 773-779

Rocha-Gaso, M.-I., March-Iborra, C., Montoya-Baides, Á., and Arnau-Vives, A., Surface generated acoustic wave biosensors for the detection of pathogens: A review, Sensors, Vol. 9, No. 7 (2009), pp. 5740-5769

Schmid, L., Weitz, D. A., and Franke, T., Sorting drops and cells with acoustics: acoustic microfluidic fluorescenceactivated cell sorter, Lab on a Chip, Vol. 14, No. 19 (2014), pp. 3710-3718

Schmid, L., Wixforth, A., Weitz, D. A., and Franke, T., Novel surface acoustic wave (SAW)-driven closed PDMS flow chamber, Microfluidics and Nanofluidics, Vol. 12, No. 1-4 (2012), pp. 229-235

Schmidt, R. V. and Coldren, L., Thin Film Acoustic Surface Waveguides on Anisotropic Media, IEEE Transactions on Sonics and Ultrasonics, Vol. 22, No. 2 (1975), pp. 115-122

Schmidt, R., Acoustic surface wave velocity perturbations in $\mathrm{LiNbO}_{3}$ by diffusion of metals, Applied Physics Letters, Vol. 27, No. 1 (1975), pp. 8-10

Schmidt, R. and Kaminow, I., Metal-diffused optical waveguides in $\mathrm{LiNbO}_{3}$, Applied Physics Letters, Vol. 25, No. 8 (1974), pp. 458-460

Seidel, H. and White, D. L., Ultrasonic surface waveguides, US Patent 3,488,602 (Jan. 1970)

Sesen, M., Alan, T., and Neild, A., Microfluidic on-demand droplet merging using surface acoustic waves, Lab on a Chip, Vol. 14, No. 17 (2014), pp. 3325-3333

Sesen, M., Devendran, C., Malikides, S., Alan, T., and Neild, A., Surface acoustic wave enabled pipette on a chip, Lab on a Chip (2017)

Shi, J., Mao, X., Ahmed, D., Colletti, A., and Huang, T. J., Focusing microparticles in a microfluidic channel with standing surface acoustic waves (SSAW), Lab on a Chip, Vol. 8, No. 2 (2008), pp. 221-223

Shibayama, K., Yamanouchi, K., Sato, H., and Meguro, T., Optimum cut for rotated Y-cut $\mathrm{LiNbO}_{3}$ crystal used as the substrate of acoustic-surface-wave filters, Proceedings of the IEEE, Vol. 64, No. 5 (1976), pp. 595-597

Shilton, R., Tan, M. K., Yeo, L. Y., and Friend, J. R., Particle concentration and mixing in microdrops driven by focused surface acoustic waves, Journal of Applied Physics, Vol. 104, No. 1 (2008), p. 014910

Shui, Y., Lin, J. M., Wu, H., Wang, N., and Chen, H., Optimization of single-phase, unidirectional transducers using three fingers per period, IEEE Transactions on Ultrasonics, Ferroelectrics, and Frequency Control, Vol. 49, No. 12 (2002), pp. 1617-1621

Silvester, P., A general high-order finite-element analysis program waveguide, IEEE Transactions on Microwave Theory and Techniques, Vol. 17, No. 4 (1969), pp. 204-210

Sinha, B. and Tiersten, H., Elastic and piezoelectric surface waves guided by thin films, Journal of Applied Physics, Vol. 44, No. 11 (1973), pp. 4831-4854 
Siqueira, M., Gatts, C., Da Silva, R., and Rebello, J., The use of ultrasonic guided waves and wavelets analysis in pipe inspection, Ultrasonics, Vol. 41, No. 10 (2004), pp. 785-797

Slobodnik, A. and Conway, E., New high-frequency high-coupling low-beam-steering cut for acoustic surface waves on $\mathrm{LiNbO}_{3}$, Electronics Letters, Vol. 6, No. 6 (1970), pp. 171-173

Sokolova, E. S., Kovalev, A. S., Timler, R., and Mayer, A. P., On the dispersion of wedge acoustic waves, Wave Motion, Vol. 50, No. 2 (2013), pp. 233-245

Szabo, T. L. and Slobodnik, A., The effect of diffraction on the design of acoustic surface wave devices, IEEE Transactions on Sonics and Ultrasonics, Vol. 20, No. 3 (1973), pp. 240-251

Tan, M. K., Tjeung, R., Ervin, H., Yeo, L. Y., and Friend, J., Double aperture focusing transducer for controlling microparticle motions in trapezoidal microchannels with surface acoustic waves, Applied Physics Letters, Vol. 95, No. 13 (2009), p. 134101

Thurston, R. N., Pierce, A. D., and Papadakis, E. P., Reference for modern instrumentation, techniques, and technology: Ultrasonic instruments and devices I: Ultrasonic instruments and devices I, Vol. 23 (1998), Academic Press

Tiersten, H. F., Linear piezoelectric plate vibrations: elements of the linear theory of piezoelectricity and the vibrations piezoelectric plates (2013), Springer

Tiersten, H., Elastic surface waves guided by thin films, Journal of Applied Physics, Vol. 40, No. 2 (1969), pp. 770-789

Tiersten, H. and Davis, R., Elastic surface waves guided by curved thin films, Journal of Applied Physics, Vol. 44, No. 5 (1973), pp. 2097-2112

Tseng, W.-K., Lin, J.-L., Sung, W.-C., Chen, S.-H., and Lee, G.-B., Active micro-mixers using surface acoustic waves on Y-cut $128 \mathrm{LiNbO}_{3}$, Journal of Micromechanics and Microengineering, Vol. 16, No. 3 (2006), p. 539

Ursell, F., Integrals with a large parameter. Several nearly coincident saddle points, Mathematical Proceedings of the Cambridge Philosophical Society, Vol. 72, Cambridge University Press (1972), pp. 49-65

Wang, W.-C. and Yang, C.-H., Antisymmetric flexural modes propagating along apex of piezoelectric wedges, Japanese Journal of Applied Physics, Vol. 46, No. 9R (2007), p. 5939

Weller, J., Crowley, J., and Giallorenzi, T., Surface acoustic waveguides on $\mathrm{LiNbO}_{3}$ formed by titanium in-diffusion, Applied Physics Letters, Vol. 31, No. 3 (1977), pp. 146-148

White, R. and Voltmer, F., Direct piezoelectric coupling to surface elastic waves, Applied Physics Letters, Vol. 7 , No. 12 (1965), pp. 314-316

Wilcox, J. Z. and Brooks, R. E., Time-Fourier transform by a focusing array of phased surface acoustic wave transducers, Journal of Applied Physics, Vol. 58, No. 3 (1985), pp. 1148-1159

Wu, T.-T., Tang, H.-T., and Chen, Y.-Y., Frequency response of a focused SAW device based on concentric wave surfaces: simulation and experiment, Journal of Physics D: Applied Physics, Vol. 38, No. 16 (2005), p. 2986

Wu, T.-T., Tang, H.-T., Chen, Y.-Y., and Liu, P.-L., Analysis and design of focused interdigital transducers, IEEE Transactions on Ultrasonics, Ferroelectrics, and Frequency Control, Vol. 52, No. 8 (2005), pp. 1384-1392

$\mathrm{Xu}, \mathrm{Z}$. and Yuan, Y. J., Implementation of guiding layers of surface acoustic wave devices: a review, Biosensors and Bioelectronics, Vol. 99 (2018), pp. 500-512

Yen, K., Bertoni, H., Oliner, A., and Markman, S., Mode coupling at a plate edge with application to acoustic ridge waveguides, Symposium on Optical and Acoustical Micro-Electronics (1975), pp. 297-309

Yen, K. and Oliner, A., The strip acoustic surface waveguide: comparison between measurement and theory, Applied Physics Letters, Vol. 28, No. 7 (1976), pp. 368-370 